\title{
ESTIMATES FROM BELOW FOR THE SPECTRAL FUNCTION AND FOR THE REMAINDER IN LOCAL WEYL'S LAW
}

\author{
DMITRY JAKOBSON AND IOSIF POLTEROVICH
}

\begin{abstract}
We obtain asymptotic lower bounds for the spectral function of the Laplacian and for the remainder in local Weyl's law on manifolds. In the negatively curved case, thermodynamic formalism is applied to improve the estimates. Key ingredients of the proof include the wave equation parametrix, a pretrace formula and the Dirichlet box principle. Our results develop and extend the unpublished thesis of A. Karnaukh [K].
\end{abstract}

\section{IntRoduction AND MAIN RESUlts}

1.1. Spectral function and Weyl's law. Let $X$ be a compact Riemannian manifold of dimension $n \geq 2$ with metric $g_{i j}$ and of volume $V$. Let $\Delta$ be the Laplacian on $X$ with the eigenvalues $0=\lambda_{0}<\lambda_{1} \leq \lambda_{2} \leq \ldots$ and the corresponding orthonormal basis $\left\{\phi_{i}\right\}$ of eigenfunctions: $\Delta \phi_{i}=\lambda_{i} \phi_{i}$.

Given $x, y \in X$, let

$$
N_{x, y}(\lambda)=\sum_{\sqrt{\lambda_{i}} \leq \lambda} \phi_{i}(x) \phi_{i}(y)
$$

be the spectral function of the Laplacian. On the diagonal $x=y$ we denote it simply $N_{x}(\lambda)$. If $N(\lambda)=\#\left\{\sqrt{\lambda_{i}} \leq \lambda\right\}$ is the eigenvalue counting function, then $N(\lambda)=\int_{X} N_{x}(\lambda) d V$. Let

$$
\sigma_{n}=\frac{2 \pi^{n / 2}}{n \Gamma(n / 2)}
$$

be the volume of the unit ball in $\mathbf{R}^{n}$. The asymptotic behavior of the spectral and the counting functions is given by ([Hor], see also [Shu $)$ :

$$
\begin{array}{rlrl}
N_{x, y}(\lambda) & =O\left(\lambda^{n-1}\right), \quad x \neq y ; & \\
N_{x}(\lambda) & =\frac{\sigma_{n}}{(2 \pi)^{n}} \lambda^{n}+R_{x}(\lambda), \quad R_{x}(\lambda)=O\left(\lambda^{n-1}\right) ; \\
N(\lambda) & =\frac{V \sigma_{n}}{(2 \pi)^{n}} \lambda^{n}+R(\lambda), \quad R(\lambda)=O\left(\lambda^{n-1}\right) .
\end{array}
$$

We refer to the asymptotics of $N_{x}(\lambda)$ as local Weyl's law, the asymptotics for $N(\lambda)$ being the usual Weyl's law for the distribution of eigenvalues.

In the present paper we focus on asymptotic lower bounds for the spectral function and for the remainder in local Weyl's law. We recall that $f_{1}(\lambda)=\Omega\left(f_{2}(\lambda)\right)$ for a function $f_{1}$ and a positive function $f_{2}$ means $\lim \sup _{\lambda \rightarrow \infty}\left|f_{1}(\lambda)\right| / f_{2}(\lambda)>0$.

Key words and phrases. Spectral function, wave kernel, Weyl's law, Anosov flow.

The first author was supported by NSERC, FQRNT, Alfred P. Sloan Foundation fellowship and Dawson fellowship. The second author was supported by NSERC and FQRNT. 
Theorem 1.1.3. Let $X$ be a compact $n$-dimensional Riemannian manifold, and let $x, y \in X$ be two points that are not conjugate along any shortest geodesic joining them. Then

$$
N_{x, y}(\lambda)=\Omega\left(\lambda^{\frac{n-1}{2}}\right) .
$$

Let us now formulate the on-diagonal counterpart of Theorem 1.1.3, Consider the heat trace asymptotics as $t \rightarrow 0^{+}$:

$$
\sum_{i} e^{-\lambda_{i} t} \sim \frac{1}{(4 \pi)^{n / 2}} \sum_{j=0}^{\infty} \int_{X} a_{j}(x) d V t^{j-\frac{n}{2}},
$$

where the local heat invariants $a_{j}(x)$ are the coefficients in the asymptotic expansion of the heat kernel (see section 2.1). We shall use the following notation. Let $\kappa_{x}=\min \left\{j \geq 1 \mid a_{j}(x) \neq 0\right\}$. If $a_{j}(x)=0$ for all $j \geq 1$ we set $\kappa_{x}=\infty$. Note that $a_{1}(x)=\frac{\tau(x)}{6}$, where $\tau(x)$ is the scalar curvature of $X$ at the point $x$.

Theorem 1.1.6. Let $X$ be an n-dimensional Riemannian manifold and $x \in X$ be an arbitrary point.

(i) If $n-2 \kappa_{x}>0$ then

$$
R_{x}(\lambda)=\Omega\left(\lambda^{n-2 \kappa_{x}}\right) .
$$

(ii) If $n-4 \kappa_{x}+1<0$ and if $x$ is not conjugate to itself along any shortest geodesic loop, then

$$
R_{x}(\lambda)=\Omega\left(\lambda^{\frac{n-1}{2}}\right) .
$$

Remark 1.1.9. If the scalar curvature $\tau(x) \neq 0$ then $\kappa_{x}=1$ and (1.1.7) becomes $R_{x}(\lambda)=\Omega\left(\lambda^{n-2}\right)$. If $n-4 \kappa_{x}+1<0$ then (1.1.8) gives a stronger estimate than (1.1.7). A bound similar to (1.1.7) holds also for the integrated remainder $R(\lambda)$ in the usual Weyl's law, see section 2.1.

Theorems 1.1 .3 and 1.1.6 (ii) are proved in sections 3.5 and 3.6 by analyzing the asymptotics of the parametrix for the wave equation. The proof of Theorem 1.1.6 (i) in section 2.1 is based on the heat kernel asymptotics. Estimate (1.1.8) in dimension two should be compared with the classical Hardy-Landau bound (and its generalization in [Sar, Proposition 3.1]) $R(\lambda)=\Omega(\sqrt{\lambda})$ for the remainder in the Gauss circle problem or, equivalently, for the remainder in Weyl's law on a 2-dimensional flat square torus (see [Sou] for the most recent improvement of such a bound). Note that on a torus $R_{x}(\lambda) \equiv R(\lambda)$.

1.2. Oscillatory error term in Weyl's law. Let us represent the local Weyl's law in a somewhat different form, introducing the oscillatory error term $R_{x}^{o s c}(\lambda)$ :

$$
N_{x}(\lambda)=\frac{1}{(4 \pi)^{n / 2}} \sum_{j=0}^{\left[\frac{n-1}{2}\right]} \frac{a_{j}(x)}{\Gamma\left(\frac{n}{2}-j+1\right)} \lambda^{n-2 j}+R_{x}^{o s c}(\lambda) .
$$

This is not an asymptotic expansion since apriori $R_{x}^{o s c}(\lambda)=O\left(\lambda^{n-1}\right)$ as the usual remainder $R_{x}(\lambda)$. The oscillatory remainder satisfies certain "logarithmic Gaussian error estimates", introduced many years ago in $[\mathrm{Br}$. This approach was adopted by physicists and the representation (1.2.1) of Weyl's law often appears in physics literature ( $\mathrm{cf}$. [B-H , (IV.13), p.37], see also [B-B]). The idea is to subtract the 
contribution of the "singularity at zero" (that we took into account in (1.1.7)) to the counting function.

Theorem 1.2.2. Let $X$ be an $n$-dimensional Riemannian manifold and let $x \in X$ be not conjugate to itself along any shortest geodesic loop. Then

$$
R_{x}^{o s c}(\lambda)=\Omega\left(\lambda^{\frac{n-1}{2}}\right) .
$$

Theorem 1.2 .2 in proved in section 3.7 .

1.3. Thermodynamic formalism. Asymptotic lower bounds (1.1.4) (1.1.7) and (1.1.8) can be improved for manifolds of negative curvature, see section 1.4. We assume that for any pair of directions $\xi, \eta$ the sectional curvature $K(\xi, \eta)$ satisfies

$$
-K_{1}^{2} \leq K(\xi, \eta) \leq-K_{2}^{2}
$$

Apart from the usual wave equation techniques (cf. [D-G], Ber, $[\mathrm{K}$ ) our method uses the thermodynamic formalism (see, for example, [Bow, $[\mathrm{P}-\mathrm{P}]$ ). Let $G^{t}$ be the geodesic flow on the unit tangent bundle $S X$ and let $E_{\xi}^{u}$ be the unstable subspace for $G^{t}, \xi \in S X$. The Sinai-Ruelle-Bowen potential is a Hölder continuous function $\mathcal{H}: S X \rightarrow \mathbf{R}$ which for any $\xi \in S X$ is defined by the formula (see [B-R], Sin2])

$$
\mathcal{H}(\xi)=\left.\left.\frac{d}{d t}\right|_{t=0} \ln \operatorname{det} d G^{t}\right|_{E_{\xi}^{u}},
$$

For any continuous function $f: S X \rightarrow \mathbf{R}$ one can define the topological pressure

$$
P(f)=\sup _{\mu}\left(h_{\mu}+\int f d \mu\right),
$$

where the supremum is taken over all $G^{t}$-invariant measures $\mu$ and $h_{\mu}$ denotes the measure-theoretic entropy of the geodesic flow (see $\mathrm{Bow}$ ). In particular $P(0)=h$, where $h$ is the topological entropy of the flow. It is well-known that for the SinaiRuelle-Bowen potential $P(-\mathcal{H})=0$ and the corresponding equilibrium measure (i.e. the measure on which the supremum is attained) is the Liouville measure $\mu_{L}$ on the unit tangent bundle:

$$
h_{\mu_{L}}=\int_{S X} \mathcal{H} d \mu_{L}
$$

1.4. Estimates for negatively curved manifolds. We now present our main results for manifolds of negative curvature.

Theorem 1.4.1. On a compact n-dimensional negatively curved manifold the spectral function $N_{x, y}(\lambda)$ satisfies for any $\delta>0$ and $x \neq y$ :

$$
N_{x, y}(\lambda)=\Omega\left(\lambda^{\frac{n-1}{2}}(\log \lambda)^{\frac{P(-\mathcal{H} / 2)}{h}-\delta}\right) .
$$

Theorem 1.4.3. The remainder in the local Weyl's law on an $n$-dimensional compact negatively curved manifold satisfies:

$$
R_{x}(\lambda)=\left\{\begin{array}{l}
\Omega\left(\lambda^{\frac{n-1}{2}}(\log \lambda)^{\frac{P(-\mathcal{H} / 2)}{h}-\delta}\right) \forall \delta>0, \quad n=2,3 ; \\
\Omega\left(\lambda^{n-2}\right), \quad n \geq 4 .
\end{array}\right.
$$

Also, in any dimension

$$
R_{x}^{\text {osc }}(\lambda)=\Omega\left(\lambda^{\frac{n-1}{2}}(\log \lambda)^{\frac{P(-\mathcal{H} / 2)}{h}-\delta}\right) \quad \forall \delta>0 .
$$


For $n=2$, a bound similar to (1.4.4) is proved in $[\mathrm{K}$, see a discussion below.

Theorems 1.4 .1 and 1.4 .3 are proved in section 5 .

Let us estimate the power $\frac{P(-\mathcal{H} / 2)}{h}$ in terms of curvatures. It was shown in [Sin1] that on an $n$-dimensional negatively curved manifold satisfying (1.3.1),

$$
(n-1) K_{2} \leq h_{\mu_{L}} \leq h \leq(n-1) K_{1}
$$

we can obtain (using the definition of pressure and (1.3.4)):

$$
P\left(-\frac{\mathcal{H}}{2}\right) \geq h_{\mu_{L}}-\int \mathcal{H} \mu_{L}+\frac{1}{2} \int \mathcal{H} d \mu_{L}=\frac{h_{\mu_{L}}}{2} \geq \frac{(n-1) K_{2}}{2}
$$

and therefore

$$
\frac{P(-\mathcal{H} / 2)}{h} \geq \frac{K_{2}}{2 K_{1}}>0 .
$$

1.5. Discussion. Remainder estimates in (1.1.2) for $N(\lambda)$ and $N_{x}(\lambda)$ are attained for round spheres and hence are sharp. One can replace $O\left(\lambda^{n-1}\right)$ by $o\left(\lambda^{n-1}\right)$ in the bounds for $R(\lambda)$ (respectively, for $R_{x}(\lambda)$ ) if the initial directions of closed geodesics (respectively, of geodesic loops at $x$ ) form a set of measure zero (for $R(\lambda)$ see [D-G], for $R_{x}(\lambda)$ see [Saf1, [S-V], section 1.8], and also [S-Z]).

Both local and integrated remainder estimates for Weyl's law were actively studied under various geometric conditions (see $[\mathrm{CdV}],[\mathrm{IV},[\mathrm{Vol}],[\mathrm{P}-\mathrm{T}]$, etc.). Of particular interest are manifolds of negative (or, more generally, nonpositive) curvature. In this case, $R_{x}(\lambda)=O\left(\lambda^{n-1} / \log \lambda\right)(\overline{\mathrm{Ber}})$. It is conjectured in Ran that on a surface of constant negative curvature $R(\lambda)=O\left(\lambda^{\frac{1}{2}+\epsilon}\right)$ for any $\epsilon>0$. In the same article it is proved that an average over $\lambda$ of $\left|N_{x, y}(\lambda)\right|$ for $x \neq y$ satisfies such an upper bound. On surfaces corresponding to quaternionic lattices $R(\lambda)=\Omega\left(\frac{\sqrt{\lambda}}{\log \lambda}\right)$ (see $[$ Hej] $)$. Hence, if there are no additional assumptions, the bound conjectured in Ran is almost the best possible.

The present paper originated as an attempt to improve the estimate $R_{x}(\lambda)=$ $\Omega(\sqrt{\lambda})$ obtained in an unpublished Princeton Ph.D. thesis $\mathrm{K}$ for surfaces of arbitrary negative curvature. The main novelty of our approach compared to [K] is the use of thermodynamic formalism. It is also noticed that the restriction to the negatively curved case considered in $[\mathrm{K}$ can be replaced by much more general assumptions, see Theorems 1.1.3 and 1.1.6 We estimate $N_{x, y}(\lambda)$ not just on the diagonal, but also for $x \neq y$. Our results apply to arbitrary dimensions, while $\mathrm{K}$ ] deals only with surfaces. Working in higher dimensions makes the analysis of the parametrix more difficult. In particular, asymptotics have different nature in high and low dimensions, see Theorem 1.4 .3

On surfaces of constant negative curvature (1.4.4) coincides with the bound in $[\mathrm{K}]$, but the techniques of thermodynamic formalism for hyperbolic flows allow us to improve the results obtained in $[\mathrm{K}$ for manifolds of variable negative curvature. We do not need the hypothesis $K_{1} / K_{2}<2$ of $\mathrm{K}$ to get a logarithmic improvement in the estimates and, moreover, we get higher powers of the logarithm. Note that the results of $\mathrm{Ran}$ ] cited above make it unlikely that in two dimensions the power of $\lambda$ in a lower bound for $N_{x}(\lambda)$ can be made greater than $1 / 2$. We would like to emphasize that the techniques of hyperbolic dynamics can be quite useful for proving spectral estimates on negatively curved manifolds (see also [Vol] where results of Bowen were applied to prove upper bounds for the remainder in Weyl's law). 
Let us conclude by mentioning that on a generic negatively curved surface it is believed that $R(\lambda)=O\left(\lambda^{\epsilon}\right)$ for any $\epsilon>0$. Such an estimate looks plausible in view of the results on spectral fluctuations, e.g. in Berry, Bo-Sch and [A-B-S]. We note the difference between the predicted upper bound for the global error term, and the lower bounds in this paper for the local remainder.

Main results of the present paper were announced in [J-P].

\section{Heat equation and wave equation techniques}

2.1. A heat kernel approach to lower bounds. In this section we prove part (i) of the Theorem 1.1.6. While the rest of the paper is based on the wave equation techniques, the contribution of the "singularity at zero" to the remainder in Weyl's law can be easier seen through the heat asymptotics.

Proof of Theorem 1.1.6 (i). For simplicity we shall assume that $\kappa_{x}=1$, if not the proof is analogous. Consider the short time asymptotic expansion of the heat kernel on the diagonal

$$
\mathcal{K}(t, x, x)=\sum_{i} e^{-\lambda_{i} t} \phi_{i}^{2}(x) \sim_{t \rightarrow 0^{+}} \frac{1}{(4 \pi)^{n / 2}} \sum_{j=0}^{\infty} a_{j}(x) t^{j-\frac{n}{2}} .
$$

The first terms are $a_{0}(x)=1, a_{1}(x)=\tau(x) / 6, \tau(x)$ is the scalar curvature at $x$. As we assumed above, $a_{1}(x) \neq 0$.

To simplify calculations we use the renormalized counting function

$$
\mathcal{N}_{x}(\mu)=\sum_{\lambda_{i} \leq \mu} \phi_{i}^{2}(x)=\frac{\sigma_{n}}{(2 \pi)^{n}} \mu^{n / 2}+\mathcal{R}_{x}(\mu),
$$

where $\mathcal{R}_{x}(\mu)$ is the renormalized remainder. Rewriting $\mathcal{K}(t, x, x)=\int_{0}^{\infty} e^{-t \mu} d \mathcal{N}_{x}(\mu)$ and integrating by parts we get:

$$
\mathcal{K}(t, x, x)=t \int_{0}^{\infty} e^{-t \mu} \mathcal{N}_{x}(\mu) d \mu=t \int_{0}^{\infty} \frac{\sigma_{n}}{(2 \pi)^{n}} e^{-t \mu} \mu^{n / 2} d \mu+t \int_{0}^{\infty} e^{-t \mu} \mathcal{R}_{x}(\mu) d \mu,
$$

Comparing this with (2.1.1) and taking into account (1.1.1) we note that the contribution of the principal term cancels out the contribution of the 0 -th heat invariant to $\mathcal{K}(t, x, x)$. Therefore,

$$
\int_{0}^{\infty} e^{-t \mu} \mathcal{R}_{x}(\mu) d \mu=a_{1}(x) t^{-\frac{n}{2}}+O\left(t^{1-\frac{n}{2}}\right), \quad t \rightarrow 0^{+} .
$$

We shall prove by contradiction that (2.1.3) implies $\mathcal{R}_{x}(\mu)=\Omega\left(\mu^{\frac{n}{2}-1}\right)$. Indeed, suppose that for any $\varepsilon>0$ there exists $z=z(\varepsilon)$ such that $\left|\mathcal{R}_{x}(\mu)\right|<\varepsilon \mu^{\frac{n}{2}-1}$ if $\mu \geq z$. We have:

$$
\left|\int_{0}^{\infty} e^{-t \mu} \mathcal{R}_{x}(\mu) d \mu\right| \leq\left|\int_{0}^{z} e^{-t \mu} \mathcal{R}_{x}(\mu) d \mu\right|+\left|\int_{z}^{\infty} e^{-t \mu} \mathcal{R}_{x}(\mu) d \mu\right|=\left|I_{1}\right|+\left|I_{2}\right| .
$$

The first term $\left|I_{1}\right|<$ const, where the constant depends on $z$ but not on $t$. The second term can be estimated by

$\left|I_{2}\right| \leq \int_{z}^{\infty} \varepsilon e^{-t \mu} \mu^{\frac{n}{2}-1} d \mu=\varepsilon t^{-\frac{n}{2}} \int_{z t}^{\infty} e^{-\nu} \nu^{\frac{n}{2}-1} d \nu \leq \varepsilon t^{-\frac{n}{2}} \int_{0}^{\infty} e^{-\nu} \nu^{\frac{n}{2}-1} d \nu=\varepsilon t^{-\frac{n}{2}} \Gamma\left(\frac{n}{2}\right)$.

Choosing $\epsilon<\frac{\left|a_{1}(x)\right|}{2 \Gamma(n / 2)}$ we get

$$
\left|\int_{0}^{\infty} e^{-t \mu} \mathcal{R}_{x}(\mu) d \mu\right| \leq \frac{\left|a_{1}(x)\right|}{2} t^{-\frac{n}{2}}
$$


as $t \rightarrow 0^{+}$, which contradicts (2.1.3). Therefore, $\mathcal{R}_{x}(\mu)=\Omega\left(\mu^{\frac{n}{2}-1}\right)$. Comparing (2.1.2) and (1.1.2) we see that it is equivalent to $R_{x}(\lambda)=\Omega\left(\lambda^{n-2}\right)$. This completes the proof of the theorem.

In order to prove a similar bound for the integrated remainder $R(\lambda)$ one should just repeat the same argument for $N(\lambda)$ instead of $N_{x}(\lambda)$ and for the heat trace instead of the heat kernel.

Remark 2.1.4. In the earlier version of the paper we proved a weaker bound $R_{x}(\lambda)=$ $\Omega\left(\lambda^{n-2 \kappa_{x}-1}\right)$ using Proposition 3.4.4. It was pointed out to us by Yu. Safarov that a better estimate (1.1.7) should be valid. He also suggested a somewhat different proof of this result based on the asymptotics of the Riesz means, cf. [Saf2].

2.2. Smoothed Fourier transform of the wave kernel. The even part of the wave kernel on $X$ satisfies

$$
e(t, x, y)=\sum_{i=0}^{\infty} \cos \left(\sqrt{\lambda_{i}} t\right) \phi_{i}(x) \phi_{i}(y)
$$

It is the fundamental solution of the wave equation

$$
\begin{aligned}
\left(\partial^{2} / \partial t^{2}-\Delta\right) e(t, x, y) & =0 \\
e(0, x, y) & =\delta(x-y), \\
(\partial / \partial t) e(0, x, y) & =0
\end{aligned}
$$

Take a smooth function $\psi \in C_{0}^{\infty}(\mathbf{R})$ such that supp $\psi \subseteq[-1,1]$, it is even and monotone decreasing on $[0,1], \psi \geq 0, \psi(0)=1$. Fix two large positive parameters $\lambda, T$ and choose a compactly supported smooth test function

$$
(1 / T) \psi(t / T) \cos (\lambda t) \text {. }
$$

Given $x, y \in M$, denote the integral in the right-hand side by

$$
k_{\lambda, T}(x, y)=\int_{-\infty}^{\infty} \frac{\psi(t / T)}{T} \cos (\lambda t) e(t, x, y) d t
$$

Substituting (2.2.1) into (2.2.4) we obtain

$$
k_{\lambda, T}(x, y)=\sum_{i=0}^{\infty} \phi_{i}(x) \phi_{i}(y) H_{\lambda, T}\left(\sqrt{\lambda_{i}}\right)
$$

where

$$
H_{\lambda, T}(r)=\int_{-\infty}^{\infty} \frac{\psi(t / T)}{T} \cos (\lambda t) \cos (r t) d t=\frac{\widehat{\psi}(T(r+\lambda))+\widehat{\psi}(T(r-\lambda))}{2}
$$

Here

$$
\widehat{\psi}(s)=\int_{-\infty}^{\infty} e^{i s \zeta} \psi(\zeta) d \zeta
$$

is the Fourier transform of $\psi$. Replacing the sum in (2.2.5) by an integral, we get the following representation of $k_{\lambda, T}(x, y)$ :

$$
\begin{aligned}
& \int_{0}^{\infty} H_{\lambda, T}(r) d N_{x, y}(r)= \\
& \int_{0}^{\infty} \frac{\widehat{\psi}(T(r+\lambda))+\widehat{\psi}(T(r-\lambda))}{2} d N_{x, y}(r)=k_{\lambda, T}(x, y)
\end{aligned}
$$

Formula 2.2.7) plays a key role in our analysis. 
On the diagonal, we shall use the following notation:

$$
\int_{0}^{\infty} H_{\lambda, T}(r) d R_{x}(r)=\widetilde{k}_{\lambda, T}(x)
$$

Note that the contribution of the main term in Weyl's law has been subtracted.

2.3. Relation between $N_{x, y}(\lambda)$ and $k_{\lambda, T}(x, y), R_{x}(\lambda)$ and $\widetilde{k}_{\lambda, T}(x)$. Assume that $T$ is either fixed or $T(\lambda) \rightarrow \infty$ as $\lambda \rightarrow \infty$. The following lemma will be used to prove Theorems 1.1.3 and 1.1.6.

Lemma 2.3.1. (i) Let $N_{x, y}(\lambda)=o\left(\lambda^{a}\right)$, a>0. Then $k_{\lambda, T}(x, y)=o\left(\lambda^{a}\right)$. (ii) Let $R_{x}(\lambda)=o\left(\lambda^{a}\right)$. Then $\widetilde{k}_{\lambda, T}(x)=o\left(\lambda^{a}\right)$.

Proof. We shall prove (i), the proof of part (ii) is analogous. Denote $\widehat{\psi}^{\prime}$ by $\Psi$. Since $\psi$ has compact support, $\Psi$ is Schwartz class. By the assumption of the lemma, for any $\epsilon>0, N_{x, y}(\lambda)<\epsilon \lambda^{a}$ for large enough $\lambda$. Consider the left hand side of (2.2.7):

$$
\int_{0}^{\infty} H_{\lambda, T}(r) d N_{x, y}(r)
$$

Taking into account (2.2.7) and integrating (2.3.2) by parts we obtain

$$
k_{\lambda, T}(x, y) \leq \frac{\epsilon T}{2} \int_{0}^{\infty}|\Psi(T(r-\lambda))| r^{a} d r+\frac{\epsilon T}{2} \int_{0}^{\infty}|\Psi(T(r+\lambda))| r^{a} d r .
$$

Since $\Psi$ is Schwartz class, the second term of (2.3.3) is $O(1)$. Changing variables in the first term of (2.3.3), we obtain

$$
\frac{\epsilon T}{2} \int_{-\lambda}^{\infty}|\Psi(T s)|(s+\lambda)^{a} d s=\frac{\epsilon \lambda^{a}}{2} \int_{-\lambda T}^{\infty}|\Psi(u)|\left(1+\frac{u}{\lambda T}\right)^{a} d u \leq C \epsilon \lambda^{a}
$$

for some constant $C>0$, where the last inequality again follows from the fact that $\Psi$ is Schwartz class. Since $\epsilon$ can be chosen arbitrarily small, we get $k_{\lambda, T}(x, y)=o\left(\lambda^{a}\right)$, and this completes the proof of the lemma.

A modification of this lemma is used to prove Theorems 1.4 .3 and 1.4 .1 .

Lemma 2.3.4. (i) Let $N_{x, y}(\lambda)=O\left(\lambda^{a}(\log \lambda)^{b}\right), a, b>0$. Then $k_{\lambda, T}(x, y)=$ $O\left(\lambda^{a}(\log \lambda)^{b}\right)$. (ii) Let $R_{x}(\lambda)=O\left(\lambda^{a}(\log \lambda)^{b}\right)$, a,b>0. Then $\widetilde{k}_{\lambda, T}(x)=O\left(\lambda^{a}(\log \lambda)^{b}\right)$.

Proof. This lemma is proved similarly to the previous one. Again, we shall prove (i), the proof of (ii) is analogous.

By the assumption of the lemma there exists a constant $C>0$ such that $N_{x, y}(\lambda)<C \lambda^{a}(\log (1+\lambda))^{b}$. Writing a representation of $k_{\lambda, T}(x, y)$ analogous to (2.3.3) we get that the second term is $O(1)$ as before, and the first term can be rewritten as

$$
\begin{gathered}
\frac{C T}{2} \int_{-\lambda}^{\infty}|\Psi(T s)|(s+\lambda)^{a}(\log (1+\lambda+s))^{b} d s= \\
\frac{C \lambda^{a}(\log \lambda)^{b}}{2} \int_{-\lambda T}^{\infty}|\Psi(u)|\left(1+\frac{u}{\lambda T}\right)^{a}\left(1+\frac{\log \left(1+\frac{1+u / T}{\lambda}\right)}{\log \lambda}\right)^{b} d u=O\left(\lambda^{a}(\log \lambda)^{b}\right) .
\end{gathered}
$$

Remark 2.3.5. Statements similar to Lemmas 2.3.1 and 2.3.4 are proved in $[\mathrm{K}$, see also [Sar, p. 226]. 
2.4. The pretrace formula. If $X$ is negatively curved it is useful to consider the fundamental solution $E(t, x, y)$ of the wave equation (2.2.2) on the universal cover $M$ of $X$. Then given $x, y \in X$, we have

$$
e(t, x, y)=\sum_{\omega \in \Gamma} E(t, x, \omega y)
$$

where the sum is taken over $\Gamma=\pi_{1}(X)$. Let $K_{\lambda, T}(x, y)$ be the analogue of $k_{\lambda, T}(x, y)$ corresponding to the wave kernel $E(t, x, y)$ on $M$ :

$$
K_{\lambda, T}(x, y)=\int_{-\infty}^{\infty} \frac{\psi(t / T)}{T} \cos (\lambda t) E(t, x, y) d t
$$

Then (2.2.7) becomes

$$
k_{\lambda, T}(x, y)=\int_{0}^{\infty} H_{\lambda, T}(r) d N_{x, y}(r)=\sum_{\omega \in \Gamma} K_{\lambda, T}(x, \omega y) .
$$

\section{Asymptotics of the Smoothed Fourier transform}

3.1. Parametrix. In this section we review the Hadamard parametrix construction for the wave equation (cf. [Ber, Prop. 27], see also [Zel]). We present it in the setting when $X$ is a manifold without conjugate points (as in the negatively curved case) and hence the parametrix is globally defined on the universal cover $M$.

We shall work in Riemannian normal coordinates centered at $x \in M$. Given $x, y \in M$, let $r=d(x, y)$ and let $E(t, x, y)$ be the fundamental solution of (2.2.2) on $M$. The parametrix for $E(t, x, y)$ is given by:

$$
E(t, x, y)=\frac{1}{\pi^{\frac{n-1}{2}}}|t| \sum_{j=0}^{\infty} u_{j}(x, y) \frac{\left(r^{2}-t^{2}\right)_{-}^{j-\frac{n-3}{2}-2}}{4^{j} \Gamma\left(j-\frac{n-3}{2}-1\right)} \quad \bmod C^{\infty},
$$

The expression (3.1.1) is understood in the sense of generalized functions [G-S. The coefficients $u_{j}(x, y)$ are the solutions of the transport equations ([Ber] $)$ along the geodesic joining $x$ and $y$ (since $X$ is negatively curved, such a geodesic on $M$ is unique). In particular $u_{0}(x, y)=g^{-1 / 2}(y)$, where $g=\sqrt{\operatorname{det} g_{i j}}$. The on-diagonal values $u_{j}(x, x)=a_{j}(x)$ are the local heat invariants, see section 2.1.

We substitute (3.1.1) into (2.2.4) to get

$$
\begin{aligned}
K_{\lambda, T}(x, y) & =\int_{-\infty}^{\infty} \frac{\psi(t / T) \cos (\lambda t)|t|}{T \pi^{\frac{n-1}{2}}} \sum_{j=0}^{J} u_{j}(x, y) \frac{\left(r^{2}-t^{2}\right)_{-}^{j-\frac{n-3}{2}-2}}{4^{j} \Gamma\left(j-\frac{n-3}{2}-1\right)} d t \\
& +E_{\lambda, T}(x, y)
\end{aligned}
$$

where $J \geq 2([n / 2]+1)+1$ and $E_{\lambda, T}(x, y)=O(1) \exp (O(T))$. ([Ber, p.266]).

3.2. Leading term cancellation in the pretrace formula. The results of sections 3.2-3.4 are essentially not new and could be deduced from Ber. We refer to [D-G, p. 46] and [Ber, p. 259] for the proper regularization of $E(t, x, x)$ at $t=0$. 
Lemma 3.2.1. The following relation holds:

$$
\begin{gathered}
(3.2 .2) \quad \int_{0}^{\infty} H_{\lambda, T}(r) d R_{x}(r)=\int_{-\infty}^{\infty} \frac{\psi(t / T) \cos (\lambda t)}{T \pi^{\frac{n-1}{2}}} \sum_{j=1}^{J} \frac{u_{j}(x, x)|t|^{2 j-n}}{4^{j} \Gamma\left(j-\frac{n-3}{2}-1\right)} d t+ \\
\sum_{g \in \Gamma \backslash\{\mathrm{Id}\}} \int_{-\infty}^{\infty} \frac{\psi(t / T) \cos (\lambda t)|t|}{T \pi^{\frac{n-1}{2}}} \sum_{j=0}^{J} \frac{u_{j}(x, \gamma x)\left(r^{2}-t^{2}\right)_{-}^{j-\frac{n-3}{2}-2}}{4^{j} \Gamma\left(j-\frac{n-3}{2}-1\right)} d t+O(1) \exp (O(T))
\end{gathered}
$$

In other words, the contribution of the 0-th term of the parametrix (3.1.1) on the diagonal into the formula (3.1.2) cancels out the contribution of the leading term in Weyl's law on the left-hand side of (2.4.3).

Proof. Substituting (1.1.1) into (1.1.2) we get:

$$
d N_{x}(r)=\frac{r^{n-1}}{2^{n-1} \pi^{n / 2} \Gamma(n / 2)}+d R_{x}(r)
$$

and hence the contribution of the leading term in Weyl's law into the left-hand side of (2.4.3) is:

$$
\int_{-\infty}^{\infty} \int_{0}^{\infty} \frac{\psi(t / T) r^{n-1} \cos (r t) d r d t}{2^{n-1} \pi^{n / 2} \Gamma(n / 2) T}
$$

The contribution of the 0 -th term of the parametrix on the diagonal to (3.1.2) is

$$
\int_{-\infty}^{\infty} \frac{\psi(t / T) \cos (\lambda t)|t|^{-n} d t}{\pi^{\frac{n-1}{2}} \Gamma\left(\frac{1-n}{2}\right) T}
$$

where $\frac{|t|^{-n}}{\Gamma\left(\frac{1-n}{2}\right)}$ is understood as a generalized function in the sense of $([\mathrm{G}-\mathrm{S}])$. If $n=2 m$ is even, $|t|^{-2 m}=t^{-2 m}$ in the sense of generalized functions and (see [Zel], G-S]):

$$
t^{-2 m}=\operatorname{Re}(t+i 0)^{-2 m}=\frac{(-1)^{m}}{(2 m-1) !} \int_{0}^{\infty} r^{2 m-1} \cos (r t) d r .
$$

Substituting this into (3.2.4) and taking into account that

$$
\Gamma\left(\frac{1}{2}-m\right)=\frac{(-1)^{m} m ! 4^{m} \sqrt{\pi}}{(2 m) !}
$$

one obtains equality between (3.2.4) and (3.2.3). If $n=2 m+1$ is odd, we have $(\mathrm{G}-\mathrm{S})$ :

$$
\frac{|t|^{-n}}{\Gamma\left(\frac{1-n}{2}\right)}=\frac{(-1)^{m} \delta^{(2 m)}(t) m !}{(2 m) !}=\frac{m !}{\pi(2 m) !} \int_{0}^{\infty} r^{2 m} \cos (r t) d r,
$$

where the second equality follows by taking the inverse Fourier transform of $\delta^{(2 m)}(t)$. Substituting (3.2.6) into (3.2.4) we again get equality between (3.2.4) and (3.2.3). Formula (3.2.2) is then a consequence of (3.1.2) and (2.4.3). This completes the proof of the lemma.

Lemma 3.2.1 implies that

$$
\tilde{k}_{\lambda, T}(x)=\sum_{\omega \in \Gamma \backslash \mathrm{Id}} K_{\lambda, T}(x, \gamma x)+\widetilde{K}_{\lambda, T}(x),
$$


where

$$
\widetilde{K}_{\lambda, T}(x)=K_{\lambda, T}(x, x)-\int_{-\infty}^{\infty} \frac{\psi(t / T) \cos (\lambda t)|t|^{-n}}{\pi^{\frac{n-1}{2}} \Gamma\left(\frac{1-n}{2}\right) T} d t .
$$

Formula (3.2.7) indicates that in order to estimate the remainder $R_{x}(\lambda)$ one has to study the leading terms in the right hand side of (3.2.7) as $\lambda \rightarrow \infty$. These are the first non-zero on-diagonal term of the parametrix and the 0 -th off-diagonal terms of the parametrix. As we shall prove below, in dimensions $n \leq 3$ the 0 -th off-diagonal term provides the principal contribution. In dimension $n \geq 4$ the lower bound (1.1.7) cannot be improved by this method.

3.3. Off-diagonal leading term. We shall consider the cases of $n$ even and $n$ odd separately. If the dimension $n=2 m$ be even, the formula 3.1.1 becomes

$$
E(t, x, y)=\frac{1}{\pi^{m-1 / 2}}|t| \sum_{j=0}^{\infty}(-1)^{j} u_{j}(x, y) \frac{\left(r^{2}-t^{2}\right)_{-}^{j-m-1 / 2}}{4^{j} \Gamma(j-m+1 / 2)} \bmod C^{\infty} .
$$

If the dimension $n=2 m+1$ is odd, the formula (3.1.1) reads

$$
E(t, x, y)=\frac{|t|}{\pi^{m}} \sum_{j=0}^{\infty}(-1)^{j} u_{j}(x, y) \frac{\left(r^{2}-t^{2}\right)_{-}^{j-m-1}}{4^{j} \Gamma(j-m)} \bmod C^{\infty} .
$$

Consider first the off-diagonal leading term in even dimensions.

Lemma 3.3.3. Let $n=2 m$. The contribution of the leading (0-th) off-diagonal term of the parametrix to (3.1.2) as $\lambda \rightarrow \infty$ is

$$
\int_{r}^{\infty} \frac{\psi(t / T) u_{0}(x, y) t \cos (\lambda t) d t}{\pi^{m-\frac{1}{2}} T \Gamma\left(\frac{1}{2}-m\right)\left(t^{2}-r^{2}\right)^{m+\frac{1}{2}}}=Q_{1} \frac{\psi(r / T) \lambda^{m-\frac{1}{2}} \sin \left(\lambda r+\phi_{m}\right)}{\sqrt{g(x, y) r^{2 m-1}} T}+O\left(\lambda^{m-\frac{3}{2}}\right),
$$

where $Q_{1}$ is some nonzero constant, $r=d(x, y)$, and $\phi_{m}=\pi / 4$ if $m$ is odd, $\phi_{m}=3 \pi / 4$ if $m$ is even.

Proof. The orders of the leading term and the remainder follow from Ber, pp. 267-268]. To compute the leading term explicitly, we rewrite the left-hand side as

$$
\begin{aligned}
& \int_{r}^{\infty} \frac{\psi(t / T) u_{0}(x, y) t \cos (\lambda t) d t}{\pi^{m-\frac{1}{2}} T \Gamma\left(\frac{1}{2}-m\right)\left(t^{2}-r^{2}\right)^{m+\frac{1}{2}}}= \\
& Q_{1}^{\prime} \int_{r}^{\infty} \frac{\psi(t / T) t \cos (\lambda t)}{T \sqrt{g(x, y)}(t+r)^{m+\frac{1}{2}}} d\left(\frac{1}{(t-r)^{m-\frac{1}{2}}}\right),
\end{aligned}
$$

where $Q_{1}^{\prime}$ is some non-zero constant. Let us integrate by parts $m$ times. In order to get the highest power of $\lambda$, all the differentiations should be applied to $\cos \lambda t$. Otherwise, the power of $\lambda$ decreases at least by 1 which implies the error estimate (the same argument is used in Lemma 3.3.5). Differentiating a cosine $m$ times one gets either a cosine or a sine, depending on the parity of $m$. This explains different phases $\phi_{m}$ for $m$ even and odd. The last step is to make a change of variables $s=t-r$ and to obtain an Erdelyi-type integral whose asymptotics is well-known (cf. [Don, Proposition A.1]).

We do not compute the constant $Q_{1}$ (as well as the constants $Q_{2}, \ldots, Q_{6}$ defined below) since their explicit value is not important. It only matters that all these constants are non-zero which one can easily check.

Remark 3.3.4. In [K, Lemma III.2] an equivalent statement is obtained for $m=1$. 
In order study the contribution of the leading off-diagonal term of (3.3.2) in (3.1.2) we transform it as follows:

$$
\begin{aligned}
& \frac{(-1)^{m} u_{0}(x, y)|t|\left(r^{2}-t^{2}\right)_{-}^{-m-1}}{\pi^{m} \Gamma(-m)}=\frac{u_{0}(x, y)|t|}{\pi^{m}}(-1)^{m} \delta^{(m)}\left(r^{2}-t^{2}\right) \\
& =\frac{u_{0}(x, y)|t|(-1)^{m}}{\pi^{m}(r+t)^{m+1}} \delta^{(m)}(r-t)=\frac{u_{0}(x, y)|t|}{\pi^{m}(r+t)^{m+1}} \delta^{(m)}(t-r)
\end{aligned}
$$

Lemma 3.3.5. Let $n=2 m+1$. The contribution of the leading (0-th) off-diagonal term of (3.3.2) in (3.1.2) is equal to

$\frac{-u_{0}(x, y)}{T \pi^{m}} \int_{-\infty}^{\infty} \frac{\psi(t / T)|t| \cos (\lambda t)}{(r+t)^{m+1}} \delta^{(m)}(t-r) d t=Q_{2} \frac{\lambda^{m} \psi(r / T) \sin \left(\lambda r+\phi_{m}\right)}{\sqrt{g(x, y)} r^{m} T}+O\left(\lambda^{m-1}\right)$,

where $Q_{2}$ is some nonzero constant, $r=d(x, y)$, and $\phi_{m}=0$ if $m$ is odd, $\phi_{m}=\pi / 2$ if $m$ is even.

Proof. This lemma follows from [Ber, p. 269]. In order to compute the principal term explicitly one has to integrate $m$ times by parts applying all derivations to $\cos (\lambda t)$. Different phases $\phi_{m}$ for $m$ even and odd appear for the same reason as in the proof of Lemma 3.3.3. The principal term is given by

$Q_{2}^{\prime} \lambda^{m} \frac{u_{0}(x, y)}{T \pi^{m}} \int_{-\infty}^{\infty} \frac{\psi(t / T)|t| \sin \left(\lambda t+\phi_{m}\right)}{(r+t)^{m+1}} \delta(t-r) d t=Q_{2} \frac{\lambda^{m} \psi(r / T) \sin \left(\lambda r+\phi_{m}\right)}{\sqrt{g(x, y)} r^{m} T}$,

where $Q_{2}, Q_{2}^{\prime}$ are some non-zero constants.

The lemmas above imply the following

Proposition 3.3.6. The integral $K_{\lambda, T}(x, y)$ defined by (2.4.2) satisfies for any $x \neq y \in M$ as $\lambda \rightarrow \infty$ :

$$
K_{\lambda, T}(x, y)=\frac{Q_{3} \lambda^{\frac{n-1}{2}} \psi(r / T)}{T \sqrt{g(x, y) r^{n-1}}} \sin \left(\lambda r+\phi_{n}\right)+O\left(\lambda^{\frac{n-3}{2}}\right) .
$$

Here $r=d(x, y), \phi_{n}=\frac{\pi}{4}(3-(n \bmod 4)), Q_{3}$ is a non-zero constant.

Proof. The proposition follows from (3.1.2) and Lemmas 3.3.3, 3.3.5, The terms in (3.1.2) for $j>0$ contribute only to the remainder in (3.3.7).

3.4. On-diagonal leading term. The on-diagonal leading term is provided by the coefficient $u_{\kappa_{x}}(x, x)=a_{\kappa_{x}}(x)$, where $\kappa_{x}$ is defined as in Theorem 1.1.6. If the scalar curvature $\tau(x) \neq 0$ then $\kappa_{x}=1$. As for the off-diagonal term, we consider even and odd dimensions separately.

Lemma 3.4.1. Let $n=2 m$ and $m \geq \kappa_{x}+1$. The contribution of the leading $\left(j=\kappa_{x}\right)$ on-diagonal term of the parametrix as $\lambda \rightarrow \infty$ in (3.2.7) is given by

$$
\int_{-\infty}^{\infty} \frac{\psi(t / T)(-1)^{\kappa_{x}} t^{2 \kappa_{x}-2 m} a_{\kappa_{x}}(x) \cos (\lambda t) d t}{4^{\kappa_{x}} \pi^{m-\frac{1}{2}} \Gamma\left(\kappa_{x}-m+\frac{1}{2}\right) T}=Q_{4} \frac{\lambda^{2 m-2 \kappa_{x}-1}}{T}+O\left(\lambda^{2 m-2 \kappa_{x}-3}\right),
$$

where $Q_{4}$ is some non-zero constant.

Proof. This lemma can be extracted from [Ber, p. 266, formula (56)]. One should expand $\psi(t / T)$ into Taylor series near 0 up to the order $2 m-2 \kappa_{x}$. Note that all terms containing odd powers of $t$ vanish after the integration. Using the fact that the Fourier transform of the generalized function $t^{-2 \alpha}$ is of order $\lambda^{2 \alpha-1}$ ([G-S $]$, we obtain the principal term and the error estimate. 
Lemma 3.4.2. Let $n=2 m+1$ and $m \geq \kappa_{x}+1$. The contribution of the the first $\left(j=\kappa_{x}\right)$ on-diagonal term of (3.3.2) to (3.2.7) is

$$
\begin{gathered}
\frac{(-1)^{m} a_{\kappa_{x}}(x)\left(m-\kappa_{x}\right) !}{4^{\kappa_{x}} \pi^{m}\left(2 m-2 \kappa_{x}\right) ! T} \int_{-\infty}^{\infty} \psi(t / T) \cos (\lambda t) \delta^{\left(2 m-2 \kappa_{x}\right)}(t) d t= \\
Q_{5} \frac{\lambda^{2 m-2 \kappa_{x}}}{T}+O\left(\lambda^{2 m-2 \kappa_{x}-2}\right),
\end{gathered}
$$

where $Q_{5}$ is some nonzero constant.

Proof. This lemma can be extracted from [Ber, p. 268]. To get the left hand side of (3.4.3) from (3.3.2) we use the first equality in (3.2.6). The asymptotics then follows from the fact that in order to get the highest power of $\lambda$, all $2 m-2 \kappa_{x}$ derivations coming from $\delta^{\left(2 m-2 \kappa_{x}\right)}(t)$ should be applied to $\cos (\lambda t)$. Note that only an even number of derivations applied to $\cos (\lambda t)$ produces a non-zero contribution, hence the orders of the principal and the error terms differ by 2 .

As consequence of these two lemmas we have the following

Proposition 3.4.4. Assume that $n>2 \kappa_{x}+1$. Then the integral $\widetilde{K}_{\lambda, T}(x)$ defined by (3.2.8) satisfies as $\lambda \rightarrow \infty$ :

$$
\widetilde{K}_{\lambda, T}(x)=\frac{Q_{6} \lambda^{n-2 \kappa_{x}-1}}{T}+O\left(\lambda^{n-2 \kappa_{x}-3}\right),
$$

where $Q_{6}$ is a non-zero constant.

Proof of Proposition 3.4.4. The proposition follows from (3.1.2) and Lemmas 3.4.1, 3.4.2. The terms in (3.1.2) for $j>\kappa_{x}$ contribute only to the remainder in (3.4.5).

3.5. Proof of Theorem 1.1.3. Let $x, y \in X$ be two arbitrary points, $r$ be the distance between them. Assume that $x$ and $y$ are not conjugate along any shortest geodesics joining them (and since the geodesics are shortest, they contain no conjugate points between $x$ and $y$ as well). In particular, this implies that there is only a finite number of shortest geodesics joining $x$ and $y$, and, moreover, there exists $\epsilon>0$ such that any other geodesic joining $x$ and $y$ has length greater than $r+\epsilon$ Mil2]. Take $T=r+\epsilon$ and consider the formula (2.2.4). The function $\psi$ is supported on $[-1,1]$, hence one can approximate the wave kernel $e(t, x, y)$ in (2.2.4) by summing up the parametrices (3.1.1) (cf. Kan, Theorem 4.3]) along geodesics of length $r$ only. Since $x$ and $y$ are not conjugate along such geodesics, the parametrices are well defined and the difference between $e(t, x, y)$ and the sum of the parametrices is a smooth function in $t$ for $t \in[0, T]$. Therefore, we can apply the results of the previous section working directly on $X$ (and not on $M$ ). In particular Lemma 3.3 .6 gives us

$$
k_{\lambda, T}(x, y)=Q_{7} \lambda^{\frac{n-1}{2}} \sin \left(\lambda r+\phi_{n}\right)+O\left(\lambda^{\frac{n-3}{2}}\right),
$$

where $Q_{3}$ is some nonzero constant. Assume for contradiction $N_{x, y}(\lambda)=o\left(\lambda^{\frac{n-1}{2}}\right)$. Set $a=\frac{n-1}{2}$ in Lemma 2.3.1. Compare Lemma 2.3.1 with (3.5.1) and fix a small number $\nu>0$. We obtain a contradiction choosing a sequence $\lambda_{k} \rightarrow \infty$, such that $\left|\sin \left(\lambda_{k} r+\phi_{n}\right)\right|>\nu$. 
3.6. Proof of the Theorem 1.1.6 (ii). The proof is similar to the proof of Theorem 1.1.3 Consider the orbit $\Gamma x$ of $x$ in the universal cover $M$. Let

$$
r=\inf _{\operatorname{Id} \neq \omega \in \Gamma} d(x, \omega x) .
$$

Since $x$ is not conjugate to itself along any shortest geodesic loop, the infimum above is attained for finitely many $\omega$-s, and there exists $\epsilon>0$ such that there are no points in $\Gamma x \backslash\{x\}$ whose distance to $x$ lies in $(r, r+\epsilon]$ ([Mil2]). Choose $T=r+\epsilon / 2$ in (2.4.2). Only the closest to $x$ lattice points contribute to the right hand side of (2.4.3). This contribution is of order $\lambda^{\frac{n-1}{2}}$. By Proposition 3.4.4 the contribution of $\omega=$ Id is of order $\lambda^{n-2 \kappa_{x}-1}$. If $n-4 \kappa_{x}+1<0, \lambda^{\frac{n-1}{2}}$ dominates and hence by the same argument as in the proof of Theorem 1.1 .3 it provides the lower bound $R_{x}(\lambda)=\Omega\left(\lambda^{\frac{n-1}{2}}\right)$ (moreover, in this case $\frac{n-1}{2}>n-2 \kappa_{x}$ and hence (1.1.7) yields a weaker bound). This completes the proof of (1.1.8).

Clearly, applicability of (1.1.7) and (1.1.8) depends on the geometry of a manifold. For instance, on flat tori (1.1.7) does not give any information since $u_{j}(x, x)=0$ for all $j \geq 1$. However, tori have no conjugate points and one can use (1.1.8).

3.7. Proof of Theorem 1.2.2, The proof of this result is analogous to the proof of Theorem 1.1.6 (ii). The condition $n-4 \kappa_{x}+1<0$ can be omitted since the sum in (1.2.1) cancels out the contributions of the on-diagonal terms of the parametrix up to the order $\left[\frac{n-1}{2}\right]$ to the pretrace formula. This can be checked by inspection of the coefficients similarly to the proof of Lemma 3.2.1. Contributions of the higher-order on-diagonal terms are negligible. Therefore, the leading contribution comes from the 0 -th off-diagonal term which is of order $\lambda^{\frac{n-1}{2}}$, and it yields the lower bound $R_{x}^{o s c}(\lambda)=\Omega\left(\lambda^{\frac{n-1}{2}}\right)$.

Remark 3.7.1. It would be interesting to understand whether one can omit the nonconjugacy condition in Theorems 1.1.3, 1.1.6 and 1.2.2. The proofs will not work since the parametrix is not well defined for conjugate points. However, our lower bounds may still hold. Moreover, estimates may get even stronger: for instance, at any point of a round sphere $R_{x}(\lambda)=\Omega\left(\lambda^{n-1}\right)$.

\section{Spectral estimates From Below on Negatively CURVED ManifoldS}

4.1. Sums over geodesic segments. Let $X$ be a negatively curved manifold with the sectional curvature satisfying (1.3.1), $M$ be the universal cover of $X$, and let $x, y \in M$. Since $X$ has no conjugate points, the parametrix is well-defined for all times on $M$.

Our strategy to strengthen Theorems 1.1.3 and 1.1.6 is as follows. Let $T$ grow with $\lambda$ and assume that we can force all the $\sin \left(\lambda r+\phi_{n}\right)$ in Proposition 3.3 .6 be of the same sign and bounded away from zero (see section 5.2). Consider the sum over geodesic segments starting at the point $x$ :

$$
S_{x, y}(T)=\sum_{r_{\omega} \leq T, \omega \in \Gamma} \frac{1}{\sqrt{g(x, \omega y) r_{\omega}^{n-1}}},
$$

where $x \neq \omega y$ for all $\omega \in \Gamma, r_{\omega}=d(x, \omega y)$ and $g=\sqrt{\operatorname{det} g_{i j}}$. The idea is to estimate (4.1.1) from below by a function going to infinity as $\lambda \rightarrow \infty$. 
Theorem 4.1.2. There exists a constant $C_{0}>0$ such that

$$
S_{x, y}(T) \geq \frac{C_{0}}{T} e^{P\left(-\frac{\mathcal{H}}{2}\right) \cdot T}
$$

as $T \rightarrow \infty$, where $P$ is the topological pressure (1.3.3) and $\mathcal{H}$ is the Sinai-RuelleBowen potential (1.3.2).

It was shown in (1.4.6) that $P\left(-\frac{\mathcal{H}}{2}\right) \geq(n-1) K_{2} / 2$, hence $S_{x, y}(T)$ grows exponentially in $\mathrm{T}$.

In the Appendix we shall prove a stronger version of (4.1.3):

$$
S_{x, y}(T) \geq C_{0} e^{P\left(-\frac{\mathcal{H}}{2}\right) \cdot T}
$$

However, a weaker estimate (4.1.3) is sufficient for the proofs of Theorems 1.4.3 and 1.4 .1 .

The proof of Theorem 4.1.2 is divided into several steps described below.

4.2. Jacobi fields and the geodesic flow. Fix a point $x \in M$ and let $\gamma(t)$ be a minimizing geodesic from $x$ to $y \in M$ such that $\gamma(0)=x, \gamma(r)=y, \gamma^{\prime}(0)=v$, where $v \in T_{x} M$ is a unit vector. Choose an orthonormal basis in $T_{x} M$ consisting of $v$ and vectors $e_{1}, e_{2}, \ldots, e_{n-1}$. To every vector $e_{j}, j=1, \ldots, n-1$, there corresponds a perpendicular Jacobi field $Y(t)$ along the geodesic $\gamma$ with the initial conditions $Y_{j}(0)=0, Y_{j}^{\prime}(0)=e_{j}$. Then (see [Ber, p. 27])

$$
g(x, y)=\frac{\sqrt{\operatorname{det}\left(Y_{j}(r), Y_{k}(r)\right)}}{r^{n-1}}
$$

Let us recall the construction of the canonical (Sasaki) metric on the tangent bundle $T M$ (see [E]). Let $\Pi: T M \rightarrow M$ be the projection map and let $\xi=(x, v) \in T M$, $\pi(\xi)=x$. Let $\mathcal{K}: T_{\xi}(T M) \rightarrow T_{x} M$ be the connection map. There exists a canonical splitting $T_{\xi} T M=T_{\xi}\left(T_{x} M\right) \oplus$ Horiz $(\xi)$ into vertical $\operatorname{Im} \mathcal{K}=T_{\xi}\left(T_{x} M\right)$ and horizontal $\operatorname{Horiz}(\xi)=\operatorname{Im} d \pi$ subspaces. For any two vectors $z, w \in T_{\xi}(T M)$ the Sasaki metric on $T M$ is defined by

$$
(z, w)_{T_{\xi}(T M)}=(d \Pi z, d \Pi w)_{T_{x} M}+(\mathcal{K} z, \mathcal{K} w)_{T_{x} M} .
$$

The induced metric on the unit tangent bundle $S M$ is also referred to as Sasaki metric.

Consider the geodesic flow $G^{t}: T M \rightarrow T M$. Then we have ([E]):

$$
Y_{j}(t)=d \Pi \circ d G^{t}\left(\tilde{e}_{j}\right), Y_{j}^{\prime}(t)=\mathcal{K} \circ d G^{t}\left(\tilde{e}_{j}\right),
$$

where $\tilde{e}_{j}=\mathcal{K}^{-1} e_{j}, j=1, \ldots, n-1$. From the definition of the Sasaki metric it follows that since $e_{j}$ are orthonormal vectors, $\tilde{e}_{j}$ are also orthonormal.

4.3. Jacobian of the geodesic flow. Consider now the unit tangent bundle $S M$. On negatively-curved manifolds there exists another natural splitting of $T_{\xi}(S M)$ into a direct sum of $D G^{t}$-invariant subspaces. It comes from the Anosov property of the geodesic flow $G^{t}$ on $S M$ :

$$
T_{\xi}(S M)=E_{\xi}^{u} \oplus E_{\xi}^{o} \oplus E_{\xi}^{s} .
$$

Here $E_{\xi}^{u}$ is the unstable subspace of dimension $(n-1), E_{\xi}^{s}$ is the stable subspace of dimension $(n-1)$, and $E_{\xi}^{o}$ is a one-dimensional subspace tangent to the flow. 
Lemma 4.3.2. There exists a universal constant $C_{1}>0$ such that

$$
\sqrt{g(x, y)} r^{\frac{n-1}{2}}<\left.C_{1} \operatorname{det} d G^{r}\right|_{E_{\xi}^{u}},
$$

where $\Pi(\xi)=x, r=d(x, y)>0$ and $E_{\xi}^{u}$ is the unstable subspace of $T_{\xi}(S M)$.

Proof. Due to (4.2.1) and (4.2.3) it remains to show that

$$
\operatorname{det}\left(d \Pi \circ d G^{r}\left(\tilde{e}_{j}\right), d \Pi \circ d G^{r}\left(\tilde{e}_{k}\right)\right) \leq C_{1}^{2}\left(\left.\operatorname{det} d G^{r}\right|_{E_{\xi}^{u}}\right)^{2}
$$

for some constant $C_{1}>0$. First, we argue that

$$
\operatorname{det}\left(d G^{r}\left(\tilde{e}_{j}\right), d G^{r}\left(\tilde{e}_{k}\right)\right)_{T_{\xi}(S M)} \geq \operatorname{det}\left(d \Pi \circ d G^{r}\left(\tilde{e}_{j}\right), d \Pi \circ d G^{r}\left(\tilde{e}_{k}\right)\right),
$$

where the scalar products on the left are taken with respect to the Sasaki metric (4.2.2). Indeed, the matrix on the left is the sum of the matrix on the right and a symmetric positive definite matrix (coming from the second term in (4.2.2) ) and hence it has a greater determinant.

Denote $\operatorname{Vert}(\xi)=T_{\xi}\left(S_{x} M\right)$ the vertical component of the canonical splitting of $S M$. Note that $\operatorname{det}\left(d G^{r}\left(\tilde{e}_{j}\right), d G^{r}\left(\tilde{e}_{k}\right)\right)_{T_{\xi}(S M)}=\left(\operatorname{Jac}_{\operatorname{Vert}(\xi)} G^{r}\right)^{2}$, where $\operatorname{Jac}_{\operatorname{Vert}(\xi)} G^{r}$ is the Jacobian of the map $G^{r}$ restricted to $\operatorname{Vert}(\xi)$ ([H-K, p. 607]). We need to prove

$$
\frac{\mathrm{Jac}_{\operatorname{Vert}(\xi)} G^{r}}{\operatorname{Jac}_{E_{\xi}^{u}} G^{r}} \leq C_{1}
$$

It is sufficient to prove this for an integer $r$. Indeed, a volume comparison argument implies $\frac{\mathrm{Jac}_{\operatorname{Vert}} \xi G^{r}}{\mathrm{Jac}_{\operatorname{Vert}} \xi G^{\text {r }}} \leq c$, where $c$ is a universal constant depending only on the curvature bounds. At the same time, it is known that $\operatorname{Jac}_{E_{\xi}^{u}} G^{t}$ is a monotone increasing function in $t$ and hence $\operatorname{Jac}_{E_{\xi}^{u}} G^{r} \geq \operatorname{Jac}_{E_{\xi}^{u}} G^{[r]}$. Taking a logarithm in (4.3.6) and writing $G^{r}=G^{1} \circ \cdots \circ G^{1}(r$ times) we get:

$$
\left|\log \operatorname{Jac}_{\operatorname{Vert}(\xi)} G^{r}-\log \operatorname{Jac}_{E_{\xi}^{u}} G^{r}\right| \leq \sum_{i=1}^{r-1}\left|\log \operatorname{Jac}_{d G^{i} \operatorname{Vert}(\xi)} G^{1}-\log \operatorname{Jac}_{E_{G^{i}}^{u}} G^{1}\right| .
$$

Let us show that the distance between the subspaces $d G^{i} \operatorname{Vert}(\xi)$ and $E_{G^{i} \xi}^{u}$ is converging to zero exponentially fast. Here distance is understood in the following sense (see [ArAv, p. 191]). For any $v \in d G^{i} \operatorname{Vert}(\xi)$ let $v=v_{u}+v_{s}$ where $v_{u} \in E_{G^{i} \xi}^{u}$, $v_{s} \in E_{G^{i} \xi}^{s}$. Set

$$
\operatorname{dist}\left(d G^{i} \operatorname{Vert}(\xi), E_{G^{i} \xi}^{u}\right)=\sup _{\|v\|=1, v \in d G^{i} \operatorname{Vert} \xi} \frac{\left\|v_{s}\right\|}{\left\|v_{u}\right\|}
$$

It then follows from the Anosov property of the geodesic flow and the results of $\mathrm{E}$, p. 456] that (4.3.7) is well defined and

$$
\operatorname{dist}\left(d G^{i} \operatorname{Vert}(\xi), E_{G^{i} \xi}^{u}\right) \leq C_{1}^{\prime} e^{-\alpha i}
$$

for some positive constants $C_{1}^{\prime}$ and $\alpha$. We now remark that $\log \mathrm{Jac}_{V} G^{1}$ depends smoothly on a subspace $V \subset T_{\eta}(S M)$, and therefore

$$
\left|\log \operatorname{Jac}_{d G^{i}} \operatorname{Vert}(\xi) G^{1}-\log \operatorname{Jac}_{E_{G^{i} \xi}^{u}} G^{1}\right| \leq C_{1}^{\prime \prime} e^{-\alpha i},
$$


where $C_{1}^{\prime \prime}$ is some positive constant that can be chosen independently of $i$ by a compactness argument $\left(G^{1}\right.$ on $S M$ is a lift of $G^{1}$ from a compact manifold $\left.S X\right)$. Hence

$$
\left|\log \operatorname{Jac}_{\operatorname{Vert}(\xi)} G^{r}-\log \operatorname{Jac}_{E_{\xi}^{u}} G^{r}\right| \leq C_{1}^{\prime \prime} \sum_{i=0}^{r-1} e^{-\alpha i} \leq \frac{C_{1}^{\prime \prime}}{1-e^{-\alpha}} .
$$

This completes the proof of the lemma.

As an immediate corollary of Lemma 4.3 .3 we get

$$
S_{x, y}(T) \geq \frac{1}{C_{1}} \sum_{r_{\omega} \leq T, \omega \in \Gamma} \frac{1}{\sqrt{\left.\operatorname{det} d G^{r_{\omega}}\right|_{E_{\xi_{\omega}}^{u}}}},
$$

where $\xi_{\omega}=\left(x, v_{\omega}\right) \in S M, v_{\omega}=\gamma_{\omega}^{\prime}(0), \gamma_{\omega}$ is the shortest geodesic joining $x$ and $\omega y$.

Denote

$Z\left(r_{\omega}, \xi_{\omega}\right)=\left.\int_{0}^{r_{\omega}} \frac{d}{d \tau}\right|_{\tau=0} \ln \operatorname{det}\left(\left.d G^{s+\tau}\right|_{E_{\xi_{\omega}}^{u}}\right) d s=\left.\ln \operatorname{det} d G^{r_{\omega}}\right|_{E_{\xi_{\omega}}^{u}}=\int_{0}^{r_{\omega}} \mathcal{H}\left(G^{s} \xi_{\omega}\right) d s$,

where $\mathcal{H}$ is the Sinai-Ruelle-Bowen potential (1.3.2). Here we used the invariance of the unstable foliation with respect to the geodesic flow. Hence,

$$
S_{x, y}(T) \geq \frac{1}{C_{1}} \sum_{r_{\omega} \leq T, \omega \in \Gamma} \exp \left(-\frac{1}{2} \int_{0}^{r_{\omega}} \mathcal{H}\left(G^{s} \xi_{\omega}\right) d s\right) .
$$

4.4. From geodesic segments to closed geodesics. Our next aim is to estimate from below the sum (4.3.8) taken over geodesic segments starting at $x$ by a sum over closed geodesics, and then apply techniques of the thermodynamic formalism. It is well-known that each conjugacy class $[\omega] \subset \Gamma$ corresponds to a unique closed geodesic $\gamma_{[\omega]}$ on $X$ of length $l_{[\omega]}$. Choose a representative $\omega_{c} \in[\omega]$ corresponding to this closed geodesic and assume that it joins $q \in M$ and $\omega_{c} q \in M$, such that $d(x, q) \leq D$ and $d\left(\omega y, \omega_{c} q\right) \leq D$, where $D$ is the diameter of $X$. Set $\xi_{[\omega]}=$ $\left(q, \gamma_{[\omega]}^{\prime}(0)\right) \in S M$. To distinguish between distances on $M$ and on $S M$ we shall write $d_{M}$ and $d_{S M}$ respectively. Here $d_{S M}$ is induced by the Sasaki metric.

Lemma 4.4.1. There exists $C_{2}>0$ such that for any $x, y \in M$ and $\omega \in \Gamma$, $d(x, \omega y) \geq D$ we have

$$
\left|Z\left(r_{\omega}, \xi_{\omega}\right)-Z\left(l_{[\omega]},\left[\xi_{[\omega]}\right]\right)\right| \leq C_{2} .
$$

Let $\gamma_{1}=\left[x_{1}, y_{1}\right]$ and $\gamma_{2}=\left[x_{2}, y_{2}\right]$ be two geodesic segments in $M$ such that $d_{M}\left(x_{1}, x_{2}\right)<D$ and $d_{M}\left(y_{1}, y_{2}\right)<D$. We shall prove the following general statement implying Lemma 4.4.1.

$$
\left|Z\left(r_{1}, \xi_{1}\right)-Z\left(r_{2}, \xi_{2}\right)\right| \leq C_{2}
$$

where $\xi_{j}=\left(x_{j}, \gamma_{j}^{\prime}(0)\right), j=1,2$ and $r_{j}=d_{M}\left(x_{j}, y_{j}\right)$.

Remark 4.4.4. It suffices to prove (4.4.3) for two geodesics starting at the same point on $M$ : apply it first to $\left(y_{1}, z_{1}\right)$ and $\left(y_{1}, z_{2}\right)$, then to $\left(y_{1}, z_{2}\right)$ and $\left(y_{2}, z_{2}\right)$.

Accordingly, let $v_{1}, v_{2} \in S M$, such that $\Pi\left(v_{1}\right)=x_{1}=x_{2}=\Pi\left(v_{2}\right)$. Let $\gamma_{v_{1}}(t)=$ $\gamma_{1}(t)$ and $\gamma_{v_{2}}(t)=\gamma_{2}(t)$ be the corresponding geodesics in $M$.

Note: to simplify notations, instead of writing $\left(\gamma(s), \gamma^{\prime}(s)\right)$ for an element of $S M$ we shall write $\gamma^{\prime}(s)$.

A key ingredient in the proof of Lemma 4.4.1 is the following 
Proposition 4.4.5. Suppose that $d_{M}\left(\gamma_{1}(t), \gamma_{2}(t)\right) \leq D$. Then there exist two constants $A_{1}>0, B_{1}>0$ such that for $0 \leq s \leq t$,

$$
d_{S M}\left(\gamma_{1}^{\prime}(s), \gamma_{2}^{\prime}(s)\right) \leq A_{1} e^{B_{1}(s-t)}
$$

4.5. Proof of Proposition 4.4.5. This proof was communicated to the authors by D. Dolgopyat. We first remark that it follows from [Schr, Lemma 2.1] that there exists $\alpha_{1}>0$ such that

$$
\operatorname{dist}\left(\gamma_{1}(s), \gamma_{2}(s)\right) \leq \alpha_{1} e^{K_{2}(s-t)},
$$

thus establishing the desired estimate on $M$.

It follows easily from the definition of $d_{S M}$ that

$$
d_{S M}\left(v_{1}, v_{2}\right) \leq \sqrt{d_{M}\left(x_{1}, x_{2}\right)^{2}+\pi^{2}}
$$

By increasing $\alpha_{1}$ if necessary, we may assume without loss of generality that (4.5.1) holds for $s \leq t+1$.

We remark that it follows easily from (4.5.1) and (4.5.2) that (4.4.6) would hold if $d_{M}\left(\gamma_{1}(s+1), \gamma_{2}(s+1)\right) \geq 1 / 3$. Accordingly, it suffices to establish (4.4.6) for such $0<s<t$ that $d_{M}\left(\gamma_{1}(s+1), \gamma_{2}(s+1)\right)<1 / 3$.

Consider now the following four points on $M$ :

$$
P_{1}=\gamma_{1}(s), P_{2}=\gamma_{2}(s), Q_{1}=\gamma_{1}(s+1), Q_{2}=\gamma_{2}(s+1) .
$$

We know that

$$
\begin{aligned}
d_{1}:= & d_{M}\left(P_{1}, P_{2}\right) \leq \alpha_{1} e^{K_{2}(s-t)}, d_{1} \leq 1 / 3 ; \\
d_{2}:= & d_{M}\left(Q_{1}, Q_{2}\right) \leq \alpha_{1} e^{K_{2}(s+1-t)}, d_{2} \leq 1 / 3 ; \\
d_{M}\left(P_{1}, Q_{1}\right)= & d_{M}\left(P_{2}, Q_{2}\right)=1 .
\end{aligned}
$$

It follows from the triangle inequality that

$$
1-d_{1} \leq d_{3}:=d_{M}\left(P_{1}, Q_{2}\right) \leq 1+d_{1} .
$$

Denote by $\gamma_{3}(s), 0 \leq s \leq d_{3}$ the geodesic segment connecting $P_{1}$ and $Q_{2}$ on $M$. Consider now the following vectors in $S M$ :

$$
w_{1}=\gamma_{1}^{\prime}(s), w_{2}=\gamma_{2}^{\prime}(s), w_{3}=\gamma_{3}^{\prime}(0), w_{4}=-\gamma_{2}^{\prime}(s+1), w_{5}=-\gamma_{3}^{\prime}\left(d_{3}\right) .
$$

The idea is to estimate $d_{S M}\left(w_{1}, w_{2}\right)$ using the triangle inequality

$$
d_{S M}\left(w_{1}, w_{2}\right) \leq d_{S M}\left(w_{1}, w_{3}\right)+d_{S M}\left(w_{2}, w_{3}\right) .
$$

We first remark that $d_{S M}\left(w_{1}, w_{3}\right)$ is equal to the angle at the vertex $P_{1}$ in the geodesic triangle $P_{1} Q_{1} Q_{2}$ with sides $d_{1}, d_{2}, d_{3}$. We compare the triangle $P_{1} Q_{1} Q_{2}$ with the planar triangle having the same sides. It follows from $d_{2}<1 / 3$, (4.5.4), elementary planar trigonometry and comparison theorem that the angle $\theta=\angle Q_{1} P_{1} Q_{2}$ is less than $\pi / 6$. Hence, $\sin \theta>3 \theta / \pi$. Therefore we have

$$
d_{S M}\left(w_{1}, w_{3}\right)=\theta<\frac{\pi \sin \theta}{3}<\frac{\pi d_{2}}{3 d_{3}}<\frac{\pi d_{2}}{2} \leq \frac{\pi \alpha_{1} e^{K_{2}}}{2} e^{K_{2}(s-t)}
$$

Here we use that $\sin \theta<d_{2} / d_{3}$ which follows from comparison with a planar triangle, and that $d_{3}>2 / 3$ which follows from the triangle inequality. It remains to estimate $d_{S M}\left(w_{2}, w_{3}\right)=d_{S M}\left(-w_{2},-w_{3}\right)$. Consider the vector $w_{6}=-\gamma_{3}^{\prime}\left(d_{3}-1\right)$. We see that

$$
d_{S M}\left(-w_{2},-w_{3}\right) \leq d_{S M}\left(-w_{2}, w_{6}\right)+d_{S M}\left(w_{6},-w_{3}\right) \leq d_{S M}\left(-w_{2}, w_{6}\right)+d_{1},
$$

so it suffices to estimate $d_{S M}\left(-w_{2}, w_{6}\right)$. 
Now,

$$
-w_{2}=G^{1}\left(w_{4}\right), \quad w_{6}=G^{1}\left(w_{5}\right),
$$

where $G^{1}$ is the time one map of the geodesic flow $G^{t}$. The distance between $w_{4}$ and $w_{5}$ is equal to the angle at $Q_{2}$ of the geodesic triangle $P_{1} Q_{2} P_{2}$ with sides $d_{3}, 1, d_{1}$. By comparing that triangle to the isometric planar triangle, one can show as in (4.5.7) that

$$
d_{S M}\left(w_{4}, w_{5}\right) \leq \frac{\pi d_{1}}{2} \leq \frac{\pi \alpha_{1}}{2} e^{K_{2}(s-t)}
$$

$\operatorname{inj}(X)$ is

Now, consider the smooth diffeomorphism $G^{1}$ restricted to the unit tangent space of the quadrilateral $P_{1} Q_{1} P_{2} Q_{2}$. By compactness it increases distances by at most a factor $\alpha_{2}$. It follows from (4.5.8) that

$$
d_{S M}\left(-w_{2}, w_{6}\right) \leq \alpha_{2} d_{S M}\left(w_{4}, w_{5}\right) \leq \frac{3 \pi \alpha_{1} \alpha_{2}}{4} e^{K_{2}(s-t)} .
$$

The inequality (4.4.6) now follows from (4.5.6), (4.5.7) and (4.5.9). This finishes the proof of the proposition.

4.6. Proof of Lemma 4.4.1, By Remark 4.4.4, in order to prove (4.4.3) it suffices to consider two vectors $\xi_{1}, \xi_{2} \in S M$ with $\pi\left(\xi_{1}\right)=\pi\left(\xi_{2}\right)$. Let $\gamma_{1}$ and $\gamma_{2}$ be geodesics as in Lemma 4.4.5. say, $\gamma_{1}=[x, \omega y]$ and $\gamma_{2}=\left[x, \omega_{c} q\right]$. Let $l_{1}, l_{2}$ be the lengths of $\gamma_{1}, \gamma_{2}$ respectively. It follows from the triangle inequality that $\left|l_{1}-l_{2}\right| \leq D$. It thus suffices to estimate the difference

$$
\left|Z\left(r_{1}, \xi_{1}\right)-Z\left(r_{2}, \xi_{2}\right)\right|=\left|\int_{0}^{r_{1}} \mathcal{H}\left(G^{s} \xi_{1}\right) d s-\int_{0}^{r_{2}} \mathcal{H}\left(G^{s} \xi_{2}\right) d s\right|
$$

Since $\mathcal{H}$ is uniformly bounded on $S X$ by compactness, and thus also on $S M$, the inequality (4.4.3) will remain true (with a different constant) if we increase or decrease the length of $\gamma_{j}$ by a uniformly bounded amount. Since $\left|l_{1}-l_{2}\right| \leq D$, we may thus assume without loss of generality that $\gamma_{j}$-s have the same length $t$.

Accordingly, to prove (4.4.2), it suffices to show that there exist $B_{2}>0$ such that for $t \geq D$,

$$
\left.\mid \int_{0}^{t}\left[\mathcal{H}\left(G^{s} \xi_{1}\right)\right)-\mathcal{H}\left(G^{s} \xi_{2}\right)\right] d s \mid \leq B_{2}
$$

It follows from the Hölder continuity of $H$ ( $\mathrm{B}-\mathrm{R}$, , Sin2] $)$ that there exist two constants $A_{2}>0, \beta>0$ such that for any $v_{1}, v_{2} \in S M$,

$$
\left|\mathcal{H}\left(v_{1}\right)-\mathcal{H}\left(v_{2}\right)\right| \leq A_{2} \cdot d_{S M}\left(v_{1}, v_{2}\right)^{\beta} .
$$

It follows from Proposition 4.4.5 that there exist two constants $A_{1}, B_{1}>0$, such that for $0 \leq s \leq t$,

$$
d_{S M}\left(G^{s} \xi_{1}, G^{s} \xi_{2}\right) \leq A_{1} e^{B_{1}(s-t)}
$$

Call the integral in (4.6.1) $I(t)$. Now,

$$
\begin{aligned}
|I(t)| & \leq \int_{0}^{t}\left|\mathcal{H}\left(G^{s} \xi_{1}\right)-\mathcal{H}\left(G^{s} \xi_{2}\right)\right| d s \leq A_{2} \int_{0}^{t} d_{S M}\left(G^{s} \xi_{1}, G^{s} \xi_{2}\right)^{\beta} d s \\
& \leq A_{2} A_{1}^{\beta} \int_{0}^{t} e^{B_{1} \beta(s-t)} d s \leq \frac{A_{2} A_{1}^{\beta}}{B_{1} \beta} .
\end{aligned}
$$


This proves (4.6.1) with $B_{2}=A_{2} A_{1}^{\beta} /\left(B_{1} \beta\right)$. The proof of Lemma 4.4.1 is complete.

4.7. Proof of Theorem 4.1.2, By Lemma 4.4.1 and (4.3.10) we have

$$
S_{x, y}(T) \geq C_{3} \sum_{\omega \in \Gamma: 3 D \leq l_{[\omega]} \leq T-2 D} \exp \left(-\frac{Z\left(l_{[\omega]}, \xi_{[\omega]}\right)}{2}\right)
$$

for some constant $C_{3}>0$. We sum over the interval $3 D \leq l_{[\omega]} \leq T-2 D$ since by triangle inequality the difference between the length of the segment and the corresponding closed geodesic is at most $2 D$, and due to the condition of Lemma 4.4 .1 we consider segments of length $\geq D$. It is a relatively rough bound. Indeed, we take into account the contribution of just one element from every conjugacy class. In the Appendix we refine this estimate taking into account the number of elements in each conjugacy class.

We now apply quite a deep fact on the equidistribution of closed geodesics for Anosov flows proved by methods of thermodynamic formalism. Using (4.7.1) we write:

$$
S_{x, y}(T) \geq \frac{C_{3}}{T} \sum_{\omega \in \Gamma: 3 D \leq l_{[\omega]} \leq T-2 D} l_{[\omega]} \exp \left(-\frac{Z\left(l_{[\omega]}, \xi_{[\omega]}\right)}{2}\right)
$$

On the other hand, the sum (4.7.2) over closed geodesics satisfies the following asymptotic relation for $T \rightarrow \infty$ : (see [Par, [P-P, (7.1)], [M-Sh, p. 109]; cf. [Ru]),

$$
\sum_{l_{[\omega]} \leq T} l_{[\omega]} \exp \left(-\frac{Z\left(l_{[\omega]}, \xi_{[\omega]}\right)}{2}\right)=\frac{C_{4}}{P(-\mathcal{H} / 2)} e^{P(-\mathcal{H} / 2) T}(1+o(1)),
$$

where

$$
C_{4}=\int_{S X} d \mu_{-\mathcal{H} / 2}
$$

Here $\mu_{-\mathcal{H} / 2}$ is the equilibrium state for $-\mathcal{H} / 2$ and $P(-\mathcal{H} / 2)$ is the pressure defined by (1.3.3). As $T \rightarrow \infty$, the contribution of closed geodesics of lengths $l_{[\omega]} \leq 3 D$ and $T-2 D \leq l_{[\omega]} \leq T$ to the sum in (4.7.3) is bounded by a constant. Therefore, (4.7.3) implies (4.1.3) and this completes the proof of Theorem 4.1.2.

\section{Proof of the main Results.}

5.1. Proof of Theorem 1.4 .1 for $n \not \equiv 3(\bmod 4)$. Given $\delta>0$, let

$$
\alpha=\frac{(1-\delta) P(-\mathcal{H} / 2)}{h} \text {. }
$$

Assume for contradiction that the bound (1.4.2) doesn't hold and thus for some $x, y \in X, x \neq y$, the off-diagonal spectral counting function $N_{x, y}(\lambda)$ satisfies the following upper bound:

$$
N_{x, y}(\lambda)=O\left(\lambda^{(n-1) / 2}(\log \lambda)^{\alpha}\right) .
$$

By Lemma 2.3.4 we conclude that there exists a constant $C_{5}>0$ such that for any $\lambda$ we have

$$
\left|k_{\lambda, T}(x, y)\right|<C_{5} \lambda^{(n-1) / 2}(\log \lambda)^{\alpha} .
$$


Using (2.4.3) we write

$$
k_{\lambda, T}(x, y)=\sum_{\omega \in \Gamma} K_{\lambda, T}(x, \omega y)
$$

We want to estimate the right-hand side from below. It follows from Proposition 3.3.6 that

$k_{\lambda, T}(x, y)=\sum_{\omega \in \Gamma, r_{\omega} \leq T} \frac{Q \lambda^{\frac{n-1}{2}}}{T} \frac{\psi\left(\frac{r_{\omega}}{T}\right)}{\sqrt{g(x, \omega y) r_{\omega}^{n-1}}} \sin \left(\lambda r_{\omega}+\phi_{n}\right)+O\left(\lambda^{\frac{n-3}{2}}\right) \exp (O(T))$.

The sum in (5.1.3) can be taken over $r_{\omega} \leq T$ since Supp $\psi=[-1,1]$. By results of Margulis ( $\mathrm{M}-\mathrm{Sh}]$ ), the number of summands in (5.1.3) is less than $C_{6} e^{h T}$ for some positive constant $C_{6}$. Since the error for each term is $O\left(\lambda^{(n-3) / 2}\right)$ by Proposition 3.3.6. for the whole sum the error is $O\left(\lambda^{\frac{n-3}{2}}\right) \exp (O(T))$.

5.2. Dirichlet box principle. We want all the terms $\sin \left(\lambda r_{\omega}+\phi_{n}\right)$ in (5.1.3) to have the same sign, and $\left|\sin \left(\lambda r_{\omega}+\phi_{n}\right)\right|$ to be bounded from below by some positive constant. Let $\left\{r_{1}, r_{2}, \ldots, r_{N_{1}}\right\}$ be all the distinct values of $r_{\omega}$ appearing in (5.1.3). It suffices to choose $\lambda$ so that for all $1 \leq j \leq N$,

$$
\left|e^{i \lambda r_{j}}-1\right|<1 / 10 \text {. }
$$

In that case all the angles $\lambda r_{j}+\phi_{n}$ are close to

$$
\phi_{n}=\pi / 4(3-n \bmod 4) .
$$

Since $n \not \equiv 3(\bmod 4), \sin \left(\lambda r_{j}+\phi_{n}\right) \approx \sin \phi_{n}$ all have the same signs and $\mid \sin \left(\lambda r_{j}+\right.$ $\left.\phi_{n}\right) \mid \geq C_{7}$ for some positive constant $C_{7}$.

To establish (5.2.1) we apply the following Lemma from [P-R] (a similar approach was also used in [R-S] to estimate error terms from below):

Lemma 5.2.2. Given $r_{1}, r_{2}, \ldots, r_{N_{1}}$ and $M_{1}>0, Y>1$ there exists $\lambda \in\left[M_{1}, M_{1} Y^{N_{1}}\right]$ such that for all $1 \leq j \leq N_{1}$,

$$
\left|e^{i \lambda r_{j}}-1\right|<1 / Y
$$

By Lemma 5.2.2 given $M_{1}>0$ we can choose $M_{1} \leq \lambda \leq M_{1} \cdot 10^{N_{1}}$ such that (5.2.1) will hold for all $1 \leq j \leq N_{1}$. We recall that $N_{1}<C_{6} e^{h T}$.

Moreover, given $\delta>0$ we can choose $\psi$ so that $\psi(x) \geq 1 / 2$ for $|x| \leq 1-\delta / 2$. Accordingly, if the radius $r_{j}$ in (5.1.3) satisfies $r_{j} \leq T(1-\delta / 2)$, then $\psi\left(r_{j} / T\right) \geq 1 / 2$. Therefore, given $M_{1}$, there exists $\lambda$ such that

$$
\ln M_{1} \leq \ln \lambda \leq \ln M_{1}+(\ln 10) C_{6} e^{h T} .
$$

It then follows from (5.1.3) that there exists $A_{3}>0$ such that

$\left|k_{\lambda, T}(x, y)\right| \geq \frac{A_{3} \lambda^{(n-1) / 2}}{T} \sum_{\omega: d_{M}(x, \omega y) \leq(1-\delta / 2) T} \frac{1}{\sqrt{g(x, \omega y) r_{\omega}^{n-1}}}+O\left(\lambda^{\frac{n-3}{2}}\right) \exp (O(T))$

In the sequel, we shall choose $T=O(\ln \ln \lambda)$. It follows that the error term in (5.2.4) is

$$
O\left(\lambda^{\frac{n-3}{2}}(\log \lambda)^{O(1)}\right), \quad \text { provided } T=O(\ln \ln \lambda)
$$


Using the estimate (4.1.3) proved in Theorem 4.1.2 we conclude from (5.2.4) and (5.2.5) that

$$
\left|k_{\lambda, T}(x, y)\right| \geq \frac{B_{3}}{T^{2}} \lambda^{\frac{n-1}{2}} e^{P(-\mathcal{H} / 2)\left(1-\frac{\delta}{2}\right) T},
$$

for some constant $B_{3}>0$.

To obtain contradiction with (5.1.2), we should find $\lambda>0, T>0$ satisfying $\lambda \geq M_{1}$ and (5.2.3)

$$
C_{5} \lambda^{(n-1) / 2}(\log \lambda)^{\alpha} \leq \frac{B_{3}}{T^{2}} \lambda^{(n-1) / 2} e^{P(-\mathcal{H} / 2)\left(1-\frac{\delta}{2}\right) T} .
$$

This translates to

$$
\begin{aligned}
\ln \ln \lambda & \leq \frac{1}{\alpha}\left[T P(-\mathcal{H} / 2)(1-\delta / 2)+\ln B_{3}-\ln C_{5}-2 \ln T\right] \\
& =\frac{h(1-\delta / 2)}{1-\delta} T+\frac{1}{\alpha}\left[\ln B_{3}-\ln C_{5}-2 \ln T\right]
\end{aligned}
$$

We can rewrite (5.2.3) as

$$
\ln \ln M_{1} \leq \ln \ln \lambda \leq h T+\ln \ln M_{1}+\ln C_{6}+\ln \ln 10 .
$$

By Lemma 5.2 .2 for any $M_{1}$ we can find some $\lambda$ satisfying (5.2.8) such that the estimate (5.2.4) holds.

Let $\beta$ be a small constant satisfying

$$
0<\beta<\frac{\delta / 2}{1-\delta}
$$

We choose $M_{1}$ in (5.2.8) to so that

$$
\ln \ln M_{1}=h \beta T \text {. }
$$

Then (5.2.8) becomes

$$
h \beta T \leq \ln \ln \lambda \leq h(1+\beta) T+\ln C_{6}+\ln \ln 10
$$

It follows that $T \approx \frac{1}{h} \ln \ln \lambda$, and thus (5.2.5) holds, implying (5.2.6).

To prove Theorem[1.4.1] it suffices to show that if we choose $T$ large enough, then (5.2.9) would imply (5.2.7). Indeed, in that case any choice of $\lambda$ satisfying (5.2.9) would automatically contradict (5.1.1), and some such choice exists by Lemma 5.2 .2 .

The largest terms in both (5.2.9) and (5.2.7) are linear in $T$, so it suffices to compare the coefficients of $T$ in those inequalities. The coefficient in (5.2.7) is equal to $\frac{h(1-\delta / 2)}{1-\delta}$ while the coefficient in (5.2.9) is equal to $h(1+\beta)$. By the choice of $\beta$, the coefficient of $T$ in (5.2.9) is smaller than that in (5.2.7). It follows that for large $T$, the right-hand side of (5.2.7) is larger than the right-hand side of (5.2.9), therefore (5.2.9) implies (5.2.7), finishing the proof of Theorem 1.4.1 for $n \not \equiv 3(\bmod 4)$.

5.3. Proof of Theorem $\mathbf{1 . 4 . 1}$ for $n \equiv 3(\bmod 4)$. The only difference between the proofs in cases $n \not \equiv 3(\bmod 4)$ and $n \equiv 3(\bmod 4)$ is that in the latter case, the angle $\phi_{n}$ in Proposition 3.3.6 is equal to zero. Accordingly, the leading term in Proposition 3.3.6 is proportional to $\sin \left(\lambda r_{\omega}\right)$ and thus we have to ensure that $\lambda r_{\omega}$ don't get too close to $\pi \mathbf{Z}$. In addition, we want to choose $\lambda$ so that $\sin \lambda r_{\omega}$ all have the same sign. Accordingly, we cannot use Lemma 5.2.2 directly and have to replace it by a different statement. 
The idea of the modification is as follows: the biggest contribution to the sum (4.1.3) in Theorem 4.1.2 comes from the terms $\omega \in \Gamma$ where $r_{\omega}=d_{M}(x, \omega y)$ is large. Indeed, for any $t>0$ every term in the sum $S_{x, y}(t)$ is not greater than 1 , therefore the number of terms gives a trivial upper bound: $S_{x, y}(t) \leq C_{6} e^{h t}$. Take

$$
A_{4}>\frac{h}{P(-\mathcal{H} / 2)(1-\delta / 2)} .
$$

and write $S_{x, y}(T)=S_{1}(T)+S_{2}(T)$, where $S_{1}(T)$ is the sum taken over all $\omega$ such that $r_{\omega}<T / A_{4}$ and $S_{2}(T)$ corresponds to

$$
T / A_{4} \leq r_{\omega} \leq T
$$

Comparing the exponents we deduce that $S_{1}(T)$ grows slower than $S_{x, y}(T)$. Indeed, $P(-\mathcal{H} / 2)(1-\delta / 2) T>h T / A_{4}$ by (5.3.1). Therefore, for $T$ large the contribution of $S_{1}(T)$ to $S_{x, y}(T)$ is negligible in the sense that $S_{x, y}(T)=S_{2}(T)(1+o(1))$.

Let $\left\{r_{1}, r_{2}, \ldots, r_{N_{2}}\right\}$ be all the $N_{2}=O\left(e^{h T}\right)$ distinct values of $r_{\omega}$ appearing in $S_{2}(T)$. Choose $Y$ satisfying

$$
Y>\frac{3 A_{4}}{\pi}
$$

By Lemma 5.2.2, given $M_{2}>0$ we can find $\mu_{1} \in\left[M_{2}, M_{2} Y^{N_{2}}\right]$ such that

$$
\operatorname{dist}\left(\mu_{1} r_{j}, 2 \pi \mathbf{Z}\right)<1 / Y, \quad 1 \leq j \leq N_{2} .
$$

The reason for choosing $Y>3 A_{4} / \pi$ will be explained below.

It suffices to find $\lambda$ such that $\sin \lambda r_{\omega}$ all have the same sign for $T / A_{4} \leq r_{\omega} \leq T$, and to estimate all those $\sin \lambda r_{\omega}$ from below. Let $2 \pi k_{j}$ be the closest multiple of $2 \pi$ to $\mu_{1} r_{j}$ in (5.3.4), and let

$$
b_{j}=\mu_{1} r_{j}-2 \pi k_{j} .
$$

We shall prove the following

Lemma 5.3.5. Let $T / A_{4} \leq r_{1}<r_{2}<\ldots<r_{N_{2}} \leq T, T \gg 1$, and let $\left|b_{j}\right| \leq 1 / Y$, $j=1, \ldots, N_{2}$. Then there exists $\mu \in[0,1]$ such that

$$
\sin \left(\mu r_{j}+b_{j}\right) \geq \frac{1}{2 T}, \quad 1 \leq j \leq N_{2} .
$$

Proof of Lemma 5.3.6. We want to find $\mu \in[0,1]$ such that

$$
\frac{1}{T} \leq \mu r_{j}+b_{j} \leq \pi-\frac{1}{T}, \quad 1 \leq j \leq m .
$$

The equality (5.3.7) is equivalent to

$$
\mu \in I_{j}:=\left[\frac{1}{T r_{j}}-\frac{b_{j}}{r_{j}}, \frac{\pi}{r_{j}}-\frac{1}{T r_{j}}-\frac{b_{j}}{r_{j}}\right] .
$$

Thus, it suffices to show that

$$
\emptyset \neq \cap_{j=1}^{m} I_{j} \cap[0,1] .
$$

The maximum $P_{1}$ of the left endpoints of $I_{j}$ is given by

$$
P_{1}=\max _{j}\left\{\frac{1}{T r_{j}}-\frac{b_{j}}{r_{j}}\right\} \leq \frac{A_{4}}{T^{2}}+\frac{A_{4}}{Y T} .
$$


On the other hand, the minimum $P_{2}$ of the right endpoints of $I_{j}$ is given by

$$
P_{2}=\min _{j}\left\{\frac{\pi}{r_{j}}-\frac{1}{T r_{j}}-\frac{b_{j}}{r_{j}}\right\} \geq \frac{\pi}{T}-\frac{A_{4}}{T^{2}}-\frac{A_{4}}{Y T} .
$$

To prove (5.3.8) it suffices to show that $P_{1}<P_{2}$. Accordingly, it suffices to show that

$$
\frac{A_{4}}{T^{2}}+\frac{A_{4}}{Y T}<\frac{\pi}{T}-\frac{A_{4}}{T^{2}}-\frac{A_{4}}{Y T}
$$

Now, it follows easily that the above inequality holds if we choose $Y>3 A_{4} / \pi$ (as in (5.3.3) ) and $T$ large enough. It follows that (5.3.8) holds, finishing the proof of Lemma 5.3.5.

The rest of the proof of the Theorem 1.4.1 for $n \equiv 3(\bmod 4)$ is the same as in the case $n \not \equiv 3(\bmod 4)$. The only difference is that in the analogue of (5.2.6) we get an extra $T$ in the denominator due to the dependence on $T$ in (5.3.6). However, this does not affect the final result since $T=O(\log \log \lambda)$ and $1 / T^{3}$ is absorbed by the factor $-\delta$ in the power of $\log \lambda$ in (1.4.2).

5.4. Proof of Theorem 1.4.3. The rate of growth of the error term on the diagonal is determined by the exponents of the leading terms in Propositions 3.3.6 (for $\omega \neq \mathrm{Id}$ ) and 3.4.4 (for $\omega=\mathrm{Id}$ ). For $n=2$ and $n=3$ the leading terms in Proposition 3.3 .6 are of order $\lambda^{(n-1) / 2}$ and grow faster than then $\widetilde{K}_{\lambda, T}(x)$.

Note that the geodesic segments considered in section 4.1 in the case of Theorem 1.4 .3 are geodesic loops. Consequently, the sum (4.1.1) should be taken over $\omega \in$ $\Gamma \backslash$ Id. We remark that the geodesic loops, and not the closed geodesics (though they are used in the proof), contribute to (4.1.1) and hence to the growth of $R_{x}(\lambda)$ (cf. section 1.5, Bogom, p.175]).

The rest of the proof of Theorem 1.4.3 is identical to that of Theorem 1.4.1. In the case $n=3$ the application of the Dirichlet principle is used appropriately as in section 5.3 .

For $n=2$ and $n=3$ (1.4.4) gives a better lower bound than (1.1.7). Note that for $n=3$ the gain is due to the logarithmic improvement guaranteed by Theorem 4.1.2. For $n \geq 4$ the "apriori" bound $R_{x}(\lambda)=\Omega\left(\lambda^{n-2}\right)$ of (1.1.7) can not be improved. This completes the proof of (1.4.4).

It remains to prove (1.4.5). We note that just as in the proof of Theorem 1.2.2 the sum in (1.2.1) cancels out the contributions of the on-diagonal $(\omega=I d)$ terms of the wave parametrix up to the order $\left[\frac{n-1}{2}\right]$ to the pretrace formula. The estimate (1.4.5) now follows from the first formula in (1.4.4) exactly in the same way as Theorem 1.2.2 is deduced from Theorem 1.1.6, see section 3.7.

Acknowledgements. The authors are grateful to D. Dolgopyat for explaining them hyperbolic dynamics leading to the results in section 4 . We would like to thank Yu. Safarov for pointing out that the bound (1.1.7) in the earlier version of the paper could be improved. We would also like to thank V. Ivrii, M. Jakobson, V. Jaksic, Y. Kannai, Ya. Pesin, M. Pollicott, L. Polterovich, P. Sarnak, R. Sharp, A. Shnirelman, M. Shubin, J. Toth and D. Wise for useful discussions. This paper was completed while the first author visited IHES and Max Planck Institute for Mathematics, and their hospitality is greatly appreciated.

\section{Appendix: PROOF of 4.1.4)}

6.1. Refining the estimate. In this section we shall prove the following 
Proposition 6.1.1. Let $S_{x, y}(T)$ be as in (4.1.1). Then

$$
S_{x, y}(T) \geq C_{0} e^{P\left(-\frac{\mathcal{H}}{2}\right) \cdot T}
$$

To prove Proposition 6.1.1 we group the terms in (4.7.1) according to conjugacy classes of $\omega \in \Gamma$. It is well-known that each conjugacy class corresponds to a unique closed geodesic on $X$. This correspondence can be defined as follows. The universal cover $M$ of $X$ can be tiled by identical copies of a fundamental domain $\Theta$ for $X$. Those copies are indexed by elements of $\Gamma=\pi_{1}(X)$. Indeed, pick a copy of $\Theta \in S$ and call it $\Theta_{e}$, where $e$ is the identity element in $\Gamma$. Then for any other copy $\widetilde{\Theta}$ there exists a unique $\omega \in \Gamma$ such that $\omega \Theta_{e}=\widetilde{\Theta}$; we then let $\widetilde{\Theta}=\Theta_{\omega}$. We thus have

$$
\omega h^{-1} \Theta_{h}=\Theta_{\omega}, \quad \forall \omega, h \in \Gamma .
$$

Given a simple closed geodesic $\gamma$ on $X$, choose its lift to $M$ passing through $\Theta_{e}$ (which we shall also call $\gamma$ ). Choose a basepoint $z \in \gamma \cap \Theta_{e}$, and let $\gamma=[z, \omega z], \omega \in$ $\Gamma$; this defines $\omega$. Now, $\gamma$ intersects other copies of the fundamental domain besides $\Theta_{e}$; denote those copies by

$$
\Theta_{h_{1}}, \Theta_{h_{2}}, \ldots, \Theta_{h_{n}}
$$

Here $h_{1}=e, h_{n}=\omega$ and $\Theta_{h_{i}}$-s are numbered consecutively along $\gamma$. One can show Mil1 that $\Gamma$ is generated by $\left\{h: \partial \Theta_{h} \cap \partial \Theta_{e} \neq \emptyset\right\}$. Let $\left\{a_{1}, a_{1}^{-1}, \ldots, a_{k}, a_{k}^{-1}\right\}$ denote a set of generators. Then each of the group elements $h_{i+1} h_{i}^{-1}, 1 \leq i \leq n-1$ is one of the generators (it maps $\Theta_{h_{i}}$ to $\Theta_{h_{i+1}}$ ). We set

$$
a_{j_{i}}:=h_{i+1} h_{i}^{-1} .
$$

Thus to a geodesic $\gamma$ we have associated an element $\omega$ and a word $w(\gamma)$ in $a_{j}$-s given by

$$
a_{j_{n}} a_{j_{n-1}} \ldots a_{j_{2}} a_{j_{1}}=\omega
$$

It is clear that

$$
n \geq l(\gamma) / \operatorname{diam}(\Theta)
$$

where $l(\gamma)$ is the length of the closed geodesic $\gamma$. In fact, it is shown in Mil1, Lemma 2 ] that (for a fixed set of generators) the ratio $l(\gamma) / L_{\Gamma}(w(\gamma))$, the denominator being the word length of $w(\gamma)$, is uniformly bounded above and below. However, we need only one side of this estimate, namely inequality (6.1.4).

If we choose a basepoint $z$ on $\gamma$ not in $\Theta_{e}$ but in adjacent fundamental domain $\Theta_{h_{1}}$, then the corresponding $\omega \in \Gamma$ becomes $\tilde{\omega}=h_{1} \omega h_{1}^{-1}$, and the word corresponding to $\tilde{\omega}$ is equal to (cf. (6.1.3)

$$
a_{j_{1}} a_{j_{n}} a_{j_{n-1}} \ldots a_{j_{2}}=a_{j_{1}} w(\gamma) a_{j_{1}}^{-1}
$$

is a cyclic shift of $w(\gamma)$. Among all such cyclic shifts, choose one (call it $w_{1}(\gamma)$ ) which will have the smallest word length (such shift is called a cyclically reduced form of $w(\gamma)$ ). We shall prove the following

Proposition 6.1.5. All cyclic shifts of a primitive cyclically reduced $w_{1}=w_{1}(\gamma)$ are different elements of $\Gamma$.

Proof of Proposition 6.1.5. It was shown by Preissman in Pre that any nontrivial commutative subgroup of $\Gamma$ is infinite cyclic. Let us now assume for 
contradiction that two cyclic shifts of $w_{1}$ coincide. We can assume without loss of generality that one shift is trivial and equals $w_{1}$ itself. So suppose that

$$
w_{1}=u_{1} u_{2}=u_{2} u_{1}
$$

for two nontrivial words $u_{1}, u_{2} \in \Gamma$. Then $u_{1}$ and $u_{2}$ generate a nontrivial commutative subgroup of $\Gamma$. Therefore, by Preissman's theorem it is cyclic and there exists $u_{3} \in \Gamma$ such that $u_{1}=u_{3}^{k}, u_{2}=u_{3}^{l}$ for some $k, l \in \mathbf{Z}$. We claim that $k$ and $l$ have the same sign, otherwise the word $w_{1}=u_{1} u_{2}$ has cancellations and is not cyclically reduced. It follows that $w_{1}=u_{3}^{k+l}$ and is therefore not primitive, which is a contradiction. This finishes the proof of the Proposition 6.1.5.

6.2. Proof of Proposition 6.1.1. We would like to get a better estimate for (4.1.1) than the one proved in section 4.7 To do that, consider a conjugacy class in $\Gamma$ and the corresponding closed geodesic $\gamma$. We associate to $\gamma$ a word $w(\gamma)$ of length $L_{\Gamma}(w(\gamma))$ as in section 6.1. By Proposition 6.1.5, to each primitive $\gamma$, there correspond $l_{\Gamma}(w(\gamma))$ cyclic shifts of this word, and by (6.1.4)

$$
L_{\Gamma}(w(\gamma)) \geq \frac{l(\gamma)}{\operatorname{diam}(\Theta)} .
$$

It is now clear from the argument of section 4.7 that to each primitive closed geodesic $\gamma$ we can associate at least $L_{\Gamma}(w(\gamma))$ segments connecting $x$ to a point on the orbit $\Gamma y$. The conclusion of Lemma 4.4.1 applies to each of these segments. Accordingly, the estimate (4.7.2) can be improved to

$$
S_{x, y}(T) \geq \frac{C_{3}}{\operatorname{diam}(\Theta)} \sum_{\omega \in \Gamma: 3 D \leq l_{[\omega]} \leq T-2 D} l_{[\omega]} \exp \left(-\frac{Z\left(l_{[\omega]}, \xi_{[\omega]}\right)}{2}\right),
$$

where $l_{[\omega]}=l(\gamma)$ is the length of the closed geodesic $\gamma$ corresponding to the group element $\omega$.

An application of 4.7.3) would complete the proof of Proposition 6.1.1 provided one can show that the sum over primitive closed geodesics is comparable to the "total" sum (4.7.3). Indeed, it easily follows that the contribution to (4.7.3) from imprimitive closed geodesics is

$$
O\left(T e^{\frac{P(-\mathcal{H} / 2) T}{2}}\right)
$$

and grows much slower than $e^{P(-\mathcal{H} / 2) T}$. Here we have used that every imprimitive geodesic corresponds to a primitive geodesic of at least twice smaller length. Therefore, the asymptotics in (4.7.3) still holds if we restrict summation to the primitive closed geodesics.

\section{REFERENCES}

[A] D. Anosov. Geodesic flows on closed Riemannian manifolds of negative curvature. Trudy Mat. Inst. Steklov. 90 (1967).

[ArAv] V. Arnold, A. Avez. Ergodic problems of classical mechanics, W.A. Benjamin, Inc., 1968. [A-B-S] R. Aurich, J. Bolte, F. Steiner. Universal signatures of quantum chaos. Phys. Rev. Lett. 73 (1994), no. 10, 1356-1359.

[B-B] R. Balian and C. Bloch. Distribution of eigenfrequencies for the wave equation in a finite domain. II. Electromagnetic field. Riemannian spaces. Ann. Physics 64 (1971), 271-307. III. Eigenfrequency density oscillations. Ann. Physics 69 (1972), 76-160.

[B-H] H. Baltes and E. Hilf. Spectra of finite systems. A review of Weyl's problem: the eigenvalue distribution of the wave equation for finite domains and its applications on the physics of small systems. Bibliographisches Institut, Mannheim-Vienna-Zurich, 1976. 116 pp. 
[Ber] P. Berard. on the wave equation on a compact riemannian manifold without conjugate points. Math. Z. 155 (1977), 249-276.

[Berry] M.V. Berry, Semiclassical theory of spectral rigidity. Proc. R. Soc. Lond. A 400 (1985), 229-251.

[Bogom] E. Bogomolny. Smoothed wavefunctions of chaotic quantum systems. Physica D 31 (1988), 169-189.

[Bo-Sch] E. Bogomolny, C Schmit. Semiclassical computations of energy levels. Nonlinearity 6 (1993), 523-547.

[Bow] R. Bowen Equilibrium states and the ergodic theory of Anosov diffeomorphisms. Lecture Notes in Math. 470. Springer, 1975.

[B-R] R. Bowen and D. Ruelle. The ergodic theory of Axiom A flows. Invent. Math. 29 (1975), no. 3, 181-202.

[Br] F.H. Brownell. An extenstion of Weyl's asymptotic law for eigenvalues. Pacific J. Math. 5 (1955), 483-499.

[Ch] I. Chavel. Riemannian geometry - a modern introduction. Cambridge Tracts in Math. 108, Cambridge Univ. Press, 1995.

[CdV] Y. Colin de Verdière. Spectre conjoint d'opérateurs pseudo-différentiels qui commutent. II. Le cas intégrable. Math. Z. 171 (1980), no. 1, 51-73.

[Don] H. Donnelly. On the wave equation asymptotics of a compact negatively curved surface. Invent. Math. 45 (1978), 115-137.

[D-G] J. Duistermaat and V. Guillemin. The spectrum of positive elliptic operators and periodic bicharacteristics. Inventiones Math. 29 (1975), 39-75.

[E] P. Eberlein. When is a geodesic flow of Anosov type? I, II. Jour. Diff. Geometry 8 (1973), $437-463,565-577$.

[G-S] I.M. Gelfand, G. E. Shilov, Generalized functions, vol. 1, Academic Press, New York, 1964.

[Hej] D. Hejhal. Selberg trace formula for PSL $(2, \mathbf{R})$, Vol. I. Lecture Notes in Math. 548, Springer, 1976.

[Hor] L. Hörmander. The spectral function of an elliptic operator. Acta Math. 121 (1968), 193218.

[Iv] V. Ivrii. Precise spectral asymptotics for elliptic operators. Lecture notes in math. 1100, Springer, 1984.

[J-P] D. Jakobson, I. Polterovich. Lower bounds for the spectral function and for the remainder in local Weyl's law on manifolds. Electron. Res. Announc. Amer. Math. Soc. 11 (2005), 71-77.

[Kan] Y. Kannai. Off diagonal short time asymptotics for fundamental solutions of diffusion equations, Commun. Partial Differ. Equations 2 (1977), no. 8, 781-830.

[H-K] A. Katok and B. Hasselblatt. Introduction to the modern theory of dynamical systems. Encyclopedia of Math. and its Apps, 54. Cambridge University Press, Cambridge, 1995.

[K] A. Karnaukh. Spectral count on compact negatively curved surfaces. Ph.D. thesis under the supervision of P. Sarnak, Princeton University (1996), 1-48.

[M-Sh] G. Margulis, On Some Aspects of the Theory of Anosov Systems. With an appendix by R. Sharp. Periodic Orbits of Hyperbolic Flows. Springer, 2004.

[Mil1] J. Milnor. A note on the curvature of the fundamental group. Jour. Diff. Geom. 2 (1967), $1-7$.

[Mil2] J. Milnor. Morse theory. Princeton University Press, 1963.

[Par] W. Parry. Equilibrium states and weighted uniform distribution of closed orbits. Dynamical Systems (College Park, MD 1986-87), Lecture Notes in Math. 1342, 617-625. Springer, 1988.

[P-P] W. Parry and M. Pollicott. Zeta functions and closed orbit structure for hyperbolic systems. Asterisque, 187-188 (1990), 1-256.

[P-T] Y. Petridis and J. Toth. The remainder in Weyl's law for Heisenberg manifolds. J. Diff. Geom. 60 (2002), no. 3, 455-483.

[P-R] R. Phillips and Z. Rudnick. The circle problem in the hyperbolic plane. J. Funct. Anal. 121 (1994), no. 1, 78-116.

[Pre] A. Preissman. Quelques propriétés globales des espaces de Riemann. Comm. Math. Helv. 15 (1943), 175-216.

[Ran] B. Randol. A Dirichlet series of eigenvalue type with applications to asymptotic estimates. Bull. London Math. Soc. 13 (1981), 309-315.

[R-S] M. Rubinstein and P. Sarnak. Chebyshev's bias. Experiment. Math. 3 (1994), no. 3, 173-197.

$[\mathrm{Ru}]$ D. Ruelle. Resonances for axiom A flows. J. Diff. Geom. 25 (1987), 99-116. 
[Saf1] Yu. Safarov. Asymptotics of a spectral function of a positive elliptic operator without a non-trapping condition. Funct. Anal. Appl. 22 (1988) no. 3, 213-223.

[Saf2] Yu. Safarov. Riesz means of the distribution function of the eigenvalues of an elliptic operator. J. Sov. Math. 49, No.5 (1990), 1210-1212 (1990); translation from Zap. Nauchn. Semin. Leningr. Otd. Mat. Inst. Steklova 163 (1987), 142-145.

[S-V] Y. Safarov and D. Vassiliev. The asymptotic distribution of eigenvalues of partial differential operators. Translations of Mathematical Monographs, 155. AMS, 1997.

[Sar] P. Sarnak. Arithmetic quantum chaos. The Schur lectures (1992) (Tel Aviv), 183-236, Israel Math. Conf. Proc., 8, 1995.

[Schr] V. Schroeder. Bounded geodesics in manifolds of negative curvature. Math. Z. 235 (2000), no. $4,817-828$.

[Sin1] Y. Sinai. The asymptotic behavior of the number of closed geodesics. AMS Transl. 73 (1968), $227-250$.

[Sin2] Y. Sinai. Gibbs measures in ergodic theory. Uspehi Mat. Nauk 27 (1972), 4(166), 21-64. Translation: Russian Math. Surveys 27 (1972), 21-69.

[Shu] M. Shubin, Pseudodifferential operators and spectral theory, Springer-Verlag, 1987.

[S-Z] C. Sogge and S. Zelditch. Riemannian manifolds with maximal eigenfunction growth. Duke Math. J. 114 (2002), no. 3, 387-437.

[Sou] K. Soundararajan. Omega bounds for the divisor and circle problems, IMRN 36 (2003), 1987-1998.

[Vol] A. Volovoy, The Hamilton flow conditions associated with Weyl's conjecture, Ann. Global Anal. Geom. vol 8, No. 2 (1990), 127-136.

[Zel] S. Zelditch, Lectures on wave invariants, in: Spectral theory and geometry, edited by B. Davies and Y. Safarov, LMS lecture note series 273, Cambridge University Press, 1999.

Department of Mathematics and Statistics, McGill University, 805 Sherbrooke Str. West, Montréal QC H3A 2K6, Canada.

E-mail address: jakobson@math.mcgill.ca

Département de mathématiques et de statistique, Université de Montréal CP 6128 succ Centre-Ville, Montréal QC H3C 3J7, Canada.

E-mail address: iossif@dms.umontreal.ca 